Check for updates

Cite this: RSC Adv., 2017, 7, 23363

\title{
Synthesis of polyphosphazenes with different side groups and various tactics for drug delivery
}

Received 21st November 2016 Accepted 10th April 2017

DOI: 10.1039/c6ra27103k

rsc.li/rsc-advances Raja Summe Ullah, Li Wang, (D) Haojie Yu, Nasir M. Abbasi, Muhammad Akram,
Zain -ul-Abdin, Muhammad Saleem, Muhammad Haroon and Rizwan Ullah Khan

Polyphosphazenes (PPZs) are hybrid polymers comprising a main chain containing nitrogen and phosphorous linked through interchanging single and double bonds, and side chains. The two chlorine atoms attached to the phosphorous of polydichlorophosphazene, which is produced by thermal ring-opening and livingcationic polymerization, are significant in the importance of polyphosphazene, as they can be easily replaced by an uncountable number of nucleophiles, resulting in hundreds of different PPZs with different properties ranging from hydrophobic to hydrophilic, fast to slow degradation, and burst to sustained drug release. More than seven hundred types of PPZs are synthesized as a result of different combinations of side groups. For applications in drug delivery, PPZs can be treated in various ways and converted into different vehicles of nano/micro size to load and release drugs. This review covers the synthesis of PPZs and their different modifications, especially focusing on the side groups and different vehicles, including microspheres, micelles, films and hydrogels, that are involved in drug-delivery applications.

\section{Introduction}

One of the greatest inventions in the field of materials is the invention of polymeric biomaterials. Natural polymers have been used as biomaterials for thousands of years, ${ }^{1}$ but scientists have started to pay attention to synthetic polymeric biomaterials only in the last century and started to use them in the last mid-century. ${ }^{2}$ The use of these biomaterials has expanded almost every field of medicine and surgery, including blood contact devices, nanomedicines and their carriers, intraocular

State Key Laboratory of Chemical Engineering, College of Chemical and Biological Engineering, Zhejiang University, Hangzhou 310027, China. E-mail: opl_wl@dial. zju.edu.cn; hjyu@zju.edu.cn; Fax: +86-571-8795-1612; Tel: +86-571-8795-3200 lenses, surgically replaced parts and tissue engineering. The polymer first used is poly(methyl methacrylate) (PMMA), which was used as an intraocular lens. ${ }^{3}$ After that, scientists started to pay more attention to the development of a variety of new biopolymers that can meet the demands of modern medical technologies. The rising fields of biotechnology, tissue engineering and medicine require such materials that are biocompatible, biodegradable and offer complete renal clearance. The commonly developed synthetic biopolymers include poly(glycolic acid), poly(lactic acid), poly(lactic-glycolic acid), poly(caprolactone), polyurethanes and polyphosphazenes. ${ }^{4}$ Among these, polyphosphazene is an auspicious biomaterial due to its bioavailability, biocompatibility, biodegradability, flexibility in its structural design and tunability of its properties. Due to this

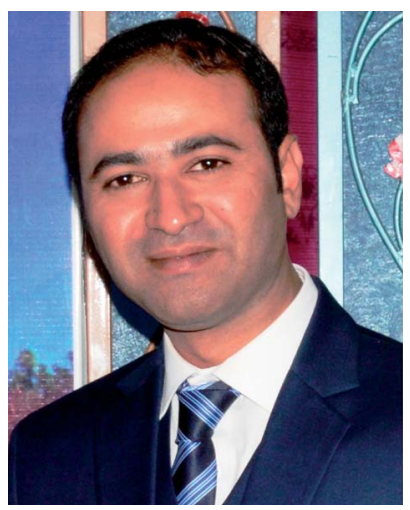

Raja Summe Ullah got his Master's degree from University of Gujrat, Pakistan. He is now a doctoral student under the supervision of Prof. Li Wang at Zhejiang University, People's Republic of China. His research interests include the synthesis of novel functional polymers mainly focusing on polyphosphazenes for drug delivery.

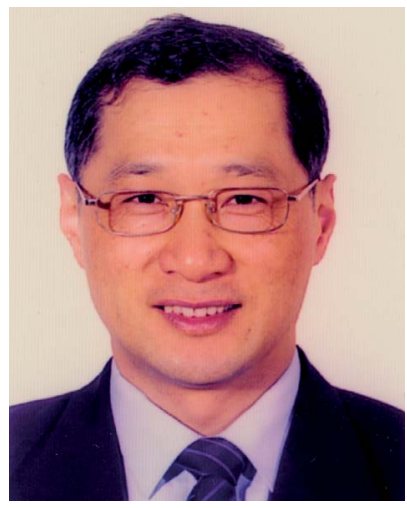

Li Wang obtained his PhD from Zhejiang University and completed postdoctoral research at Waterloo University, Waterloo, Canada. He was a Visiting Professor at University of Washington, Seattle, WA, USA. He currently holds the title of professor and doctor tutor at Zhejiang University. His research interests mainly focus on functional polymers, nanomaterials, and novel catalysts for olefin polymerization. 
<smiles>ClP1(Cl)=NP(Cl)(Cl)=NP(Cl)(Cl)=N1</smiles>

$\mathbf{a}$

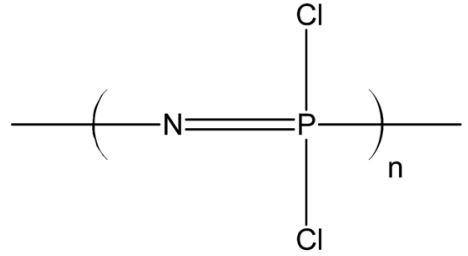

b
Fig. 1 (a) Hexachlorocyclotriphosphazene (HCCP), (b) polydichlorophosphazene (PDCP).

all-in-one presentation of polyphosphazene, it is a promising class of biopolymer. Hundreds of different kinds of polyphosphazenes (PPZs) with different characteristics and behaviors have been synthesized, most of which are biodegradable and can be used for biomedical applications. PPZs are a class of hybrid organic-inorganic polymers. The main chain of PPZs comprises phosphorous and nitrogen, which are bound with each other through alternating single and double bonds. The most interesting thing in the formation of polyphosphazene is the presence of two chlorine atoms on the phosphorous, as shown in Fig. 1. These chlorine atoms can be easily replaced by numerous side groups, which is very rare in other polymers. As a result, the number of PPZs with different side groups and a wide range of properties is growing day by day..$^{5,6}$

A wide variety of different organic molecules can act as nucleophiles for the substitution of chlorine in polydichlorophosphazene (PDCP), which broadens the spectrum of PPZs with different properties. Initially, the synthesized PPZs were not degradable in water because they formed an insoluble rubber-type material due to cross-linking of chains, so they could not be utilized for biological purposes. Later on, the introduction of specific side groups, such as amino acid esters, alkoxy, imidazole and amines, to the main chain resulted in hydrolytically sensitive and degradable PPZs. The non-toxic degradation products of PPZs opened new era of their biomedical application, including drug delivery and tissue engineering. Furthermore, the degradation of PPZs can be regulated by the substitution of hydrophilic and hydrophobic groups, or the combination of both groups in appropriate ratios. ${ }^{7}$ The degradation rate can also be controlled by external stimuli, such as temperature, radiation, $\mathrm{pH}$ of the degradation medium and degradation product solubility. Many researchers have investigated the biocompatibility of PPZs both in vivo and in vitro by different methods. ${ }^{7-10}$ Nowadays, the use of PPZs in drug-delivery applications, ${ }^{11}$ especially proteins and anti-cancer drug-delivery, has become a significant focus ${ }^{12-14}$ The synthetic flexibility of PPZs offers the possibility of designing polymers with the required properties and degradation rates, which are valuable for the development of various carriers to deliver drugs. This paper reviews the effects of various side groups attached through different linkage sites on the properties of resultant PPZs and their use in different approaches, such as drug delivery.

\section{Synthesis of polyphosphazenes}

PPZs are normally synthesized from two main raw materials: hexachlorocyclotriphosphazene $\left(\mathrm{N}_{3} \mathrm{P}_{3} \mathrm{Cl}_{6}\right)$, which is used in thermal ring-opening polymerization, and phosphoranimine $\left(\mathrm{Cl}_{3} \mathrm{P}=\mathrm{NSiMe}_{3}\right)$, which is used in living cationic polymerization. No matter which starting material is used, the intermediate stage of all the PPZs prepared by these two processes is PDCP. The synthesis of non-cross-linked PDCP is the key step for the fruitful preparation of PPZs. ${ }^{15}$ Cross-linking of PDCP due to the presence of moisture or air (mostly water vapours) may result in swelling and insolubility in any solvent. Many researchers have used different methods to synthesize PDCP. ${ }^{16,17}$

\subsection{Thermal ring-opening polymerization}

The most commonly used route to synthesize PPZs is thermal ring-opening polymerization (TROP), which is extensively employed for the ring-opening process of hexachlorocyclotriphosphazene (HCCP) to synthesize PDCP., Stokes was the pioneer of using this method in 1897, but the PDCP he synthesized was cross-linked and could not be dissolved in any solvent. This process gained attention in 1965 when Allcock and co-workers successfully synthesized non-

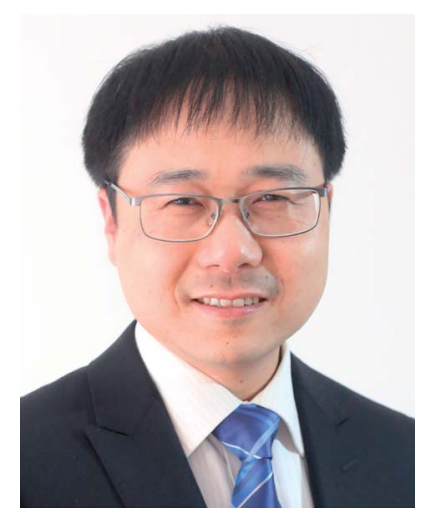

Haojie $\mathrm{Yu}$ is an associate professor and doctor tutor at the College of Chemical and Biological Engineering, Zhejiang University, China. He received his PhD degree and did postdoctoral research at Zhejiang University. He served as a visiting associate researcher at University of California, Irvine, USA. His research interests mainly focus on novel functional polymers, nanocomposites, and coatings.

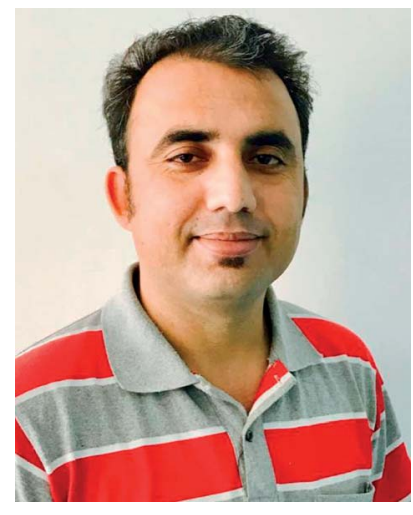

Mr Muhammad Haroon is currently a doctoral student in Zhejiang University, Hangzhou China. He has done his master degree in organic chemistry at Gomal University Dera Ismail Khan, Pakistan. His research interests are functional polymers, especially starch derivatives. 
1) Initiator formation

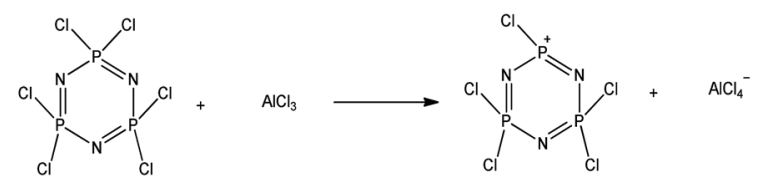

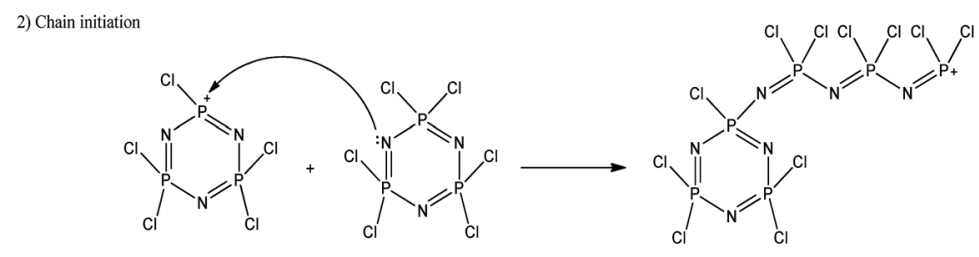

3) Propagation<smiles>ClP(Cl)(=NP(Cl)(Cl)=NP(Cl)(Cl)=NP(Cl)(Cl)=NP1(Cl)=NP(Cl)(Cl)=NP(Cl)(Cl)=N1)N=P(Cl)(Cl)N=P(Cl)(Cl)N=P(Cl)(Cl)N=P1(Cl)N=P(Cl)(Cl)N=P(Cl)(Cl)N=P1(Cl)Cl</smiles>

4) Chain termination

4.1) Termination by catalyst<smiles>ClP(Cl)(Cl)=NP(Cl)(Cl)=NP(Cl)(Cl)=NP(Cl)(Cl)=NP(Cl)(Cl)=NP(Cl)(Cl)=NP(Cl)(Cl)=NP1(Cl)=NP(Cl)(Cl)=NP(Cl)(Cl)=N1</smiles>

4.2) Termination by monomer<smiles>ClP(Cl)(=NP(Cl)(Cl)=NP(Cl)(Cl)=NP(Cl)(Cl)=NP(Cl)(Cl)=NP1(Cl)=NP(Cl)(Cl)=NP(Cl)(Cl)=N1)N=P(Cl)(Cl)N=P(Cl)(Cl)N=P(Cl)(Cl)N=P(Cl)(Cl)N=P1(Cl)N=P(Cl)(Cl)N=P(Cl)(Cl)N=P1(Cl)Cl</smiles>

Scheme 1 Reaction mechanism of ring-opening polymerization. ${ }^{27}$

crosslinked PDCP that showed good solubility in various organic solvents. ${ }^{18}$ This process involves the heating of HCCP at high temperature under vacuum and in the presence of a suitable catalyst to produce linear-chain PDCP. The two chlorine atoms present on each phosphorous are highly reactive and can be easily replaced by suitable nucleophilic groups to synthesize a variety of PPZs. ${ }^{18-20}$ If the heating temperature is increased to $300^{\circ} \mathrm{C}$, PDCP is converted into cross-linked inorganic "rubber", which cannot be used further. ${ }^{21}$ This process can yield highmolecular-weight polyphosphazenes, but it is highly sensitive

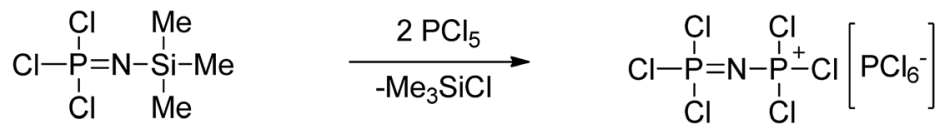

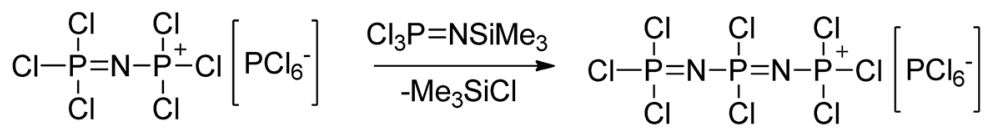

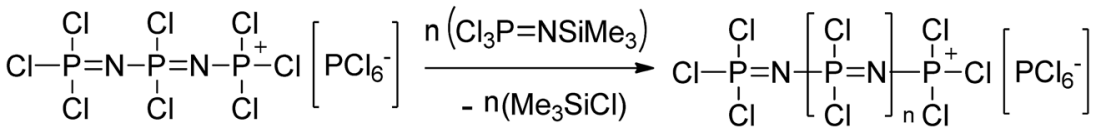

Scheme 2 Mechanism of living cationic polymerization. The "living" end can continue the process to increase the chain length. ${ }^{17}$ 

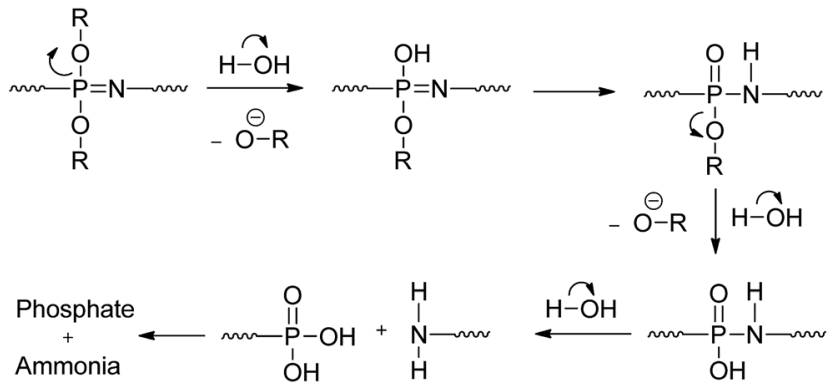

Scheme 3 Hydrolytic degradation of side groups and polyphosphazene chain. ${ }^{48}$

to the purity of monomer as well as moisture and air. Thus, the reaction proceeds under high vacuum, and the HCCP is recrystallized and sublimed before use. The yield of reaction was not so good and was normally between $40-60 \%$, which compelled the researchers to use different catalysts, such as $\mathrm{AlCl}_{3}, \mathrm{BCl}_{3}$, and $\mathrm{R}_{3} \mathrm{Si}\left(\mathrm{CHB}_{11} \mathrm{X}_{11}\right)$, to enhance the yield. ${ }^{22-26}$ Scheme 1 provides general mechanism that is followed in thermal ring-opening polymerization. ${ }^{27}$ This process of ring opening can proceed either in vacuum ${ }^{28}$ or in high-boiling solvents. ${ }^{29-31}$ Another weakness of this process is that there is little control over molecular weights and polydispersities (PDI) are normally high, ${ }^{5,32}$ but this can be overcome by careful handling and strict control over the reaction conditions and initial concentrations of HCCP and catalyst. ${ }^{22}$ This process is advantageous for the large-scale production of PDCP up to kilogram level, including the stabilization of PDCP in diglyme. ${ }^{28}$

\subsection{Living cationic polymerization}

PPZs can be synthesized by an alternative method that does not involve the use of high temperature, namely, living cationic polymerization (LCP). ${ }^{33-35}$ Allcock and co-workers succeeded in 1995 to synthesize polyphosphazene from another monomer, trichloro-(trimethylsilyl)-phosphoarnimine $\quad\left(\mathrm{Cl}_{3} \mathrm{P}=\mathrm{NSiMe}_{3}\right)$, using $\mathrm{PCl}_{5}$ as initiator. ${ }^{33}$ This process is mostly suitable for the synthesis of a wide variety of polymers, including polyphosphazene block copolymers. Examples of such polymers include polyphosphazene-block-polyphosphazene copolymers, ${ }^{33}$ poly(ethylene oxide)-block-polyphosphazene copolymers ${ }^{36,37}$ and polystyrene-block-polyphosphazene copolymers. ${ }^{38,39}$ Similarly, other structures have also been designed, such as tri-block copolymers and tri-armed, star-shaped and telecholic PPZs using ambient temperature conditions. ${ }^{36,40-42}$ Similar to ringopening polymerization, the properties of synthesized polymers depend on the molecular weight and nature of side groups. Another important advantage of this method is its molecular weight control and narrow polydispersity. ${ }^{43}$ The general mechanism of living cationic polymerization is given in Scheme $2 .{ }^{17}$

\section{Types of linkages in polyphosphazenes}

Different substituents have been linked to the main chain of PPZs, but almost all the substituents use three different kinds of combinations for the attachment to the main-chain phosphorous: (a) two substituents linked to phosphorous through nitrogen atoms, (b) two substituents linked to phosphorous through oxygen atoms, and (c) one linked through oxygen, and the other through nitrogen at the same time. Different substituents attached through these three types of linkages have different effects on the properties of PPZs. Side groups are very important in determining two major properties of PPZs: biodegradability and biocompatibility. They can alter the hydrophilic and hydrophobic nature of polymers, $T_{\mathrm{g}}$ values, physiology and, ultimately, their degradation behavior, which is very important for their application in drug delivery. Degradation of PPZs is mostly due to the difference in the polarities of phosphorous and other linking atoms-mostly oxygen and nitrogen-as well as their hydrophobicity. This difference in polarities permits nucleophilic attack by water molecules to

(A)

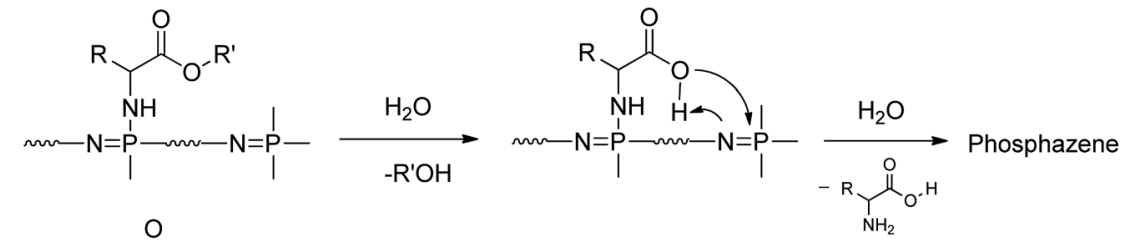

(B)

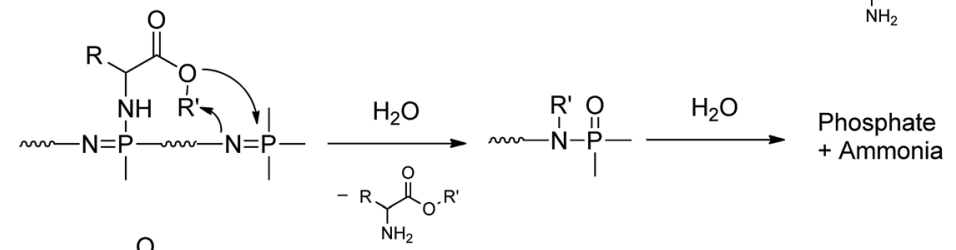

(C)

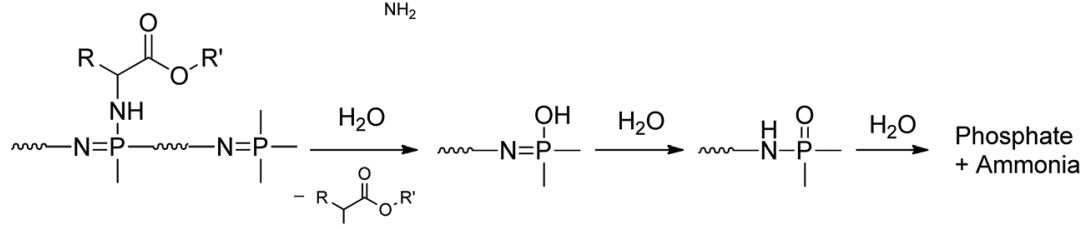


Table 1 Different side groups linked through the nitrogen of PPZ, degradation rates and $T_{\mathrm{g}}$ values ${ }^{a}$

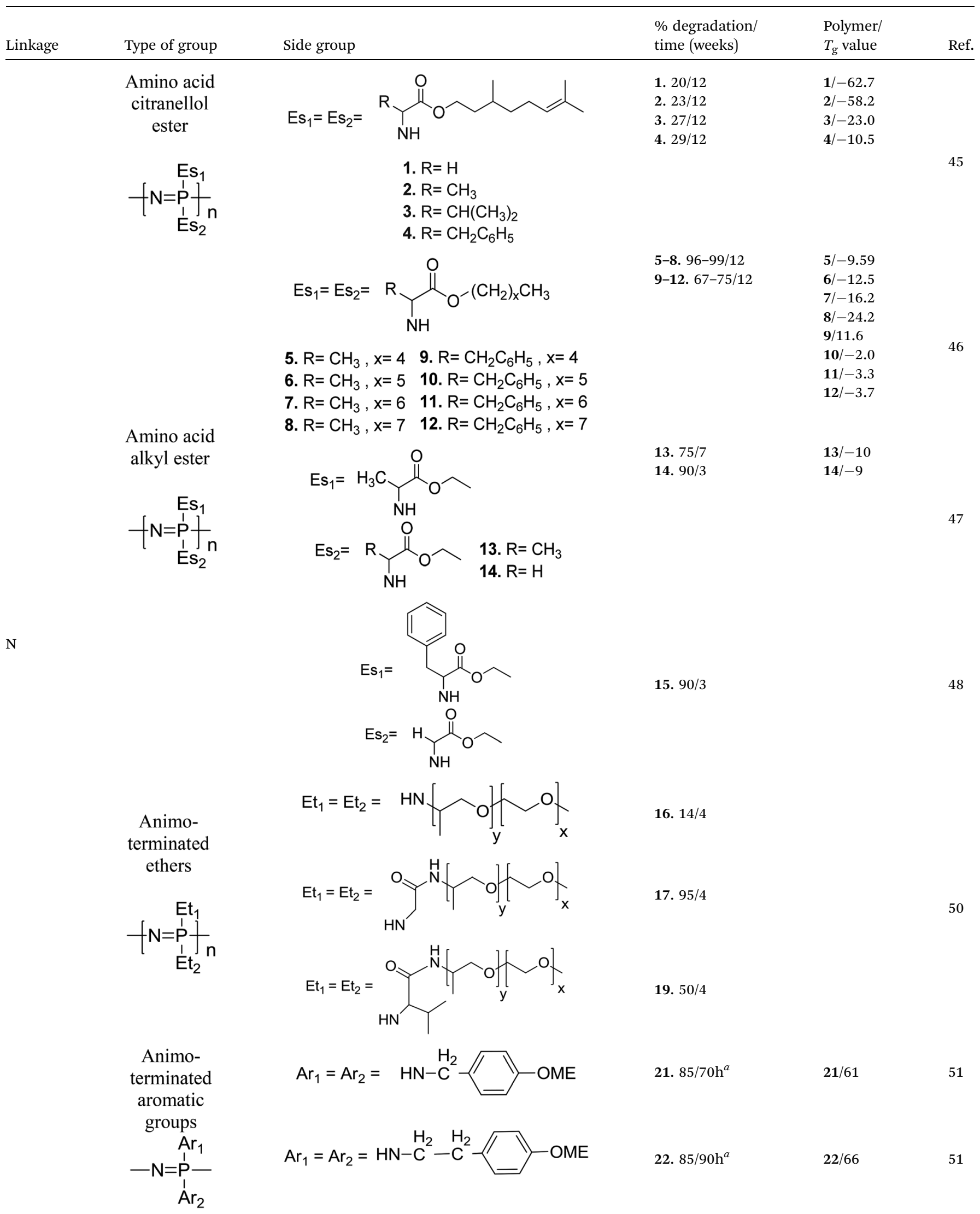

\footnotetext{
${ }^{a}$ Based on drug release profile of microspheres prepared from polymers.
} 

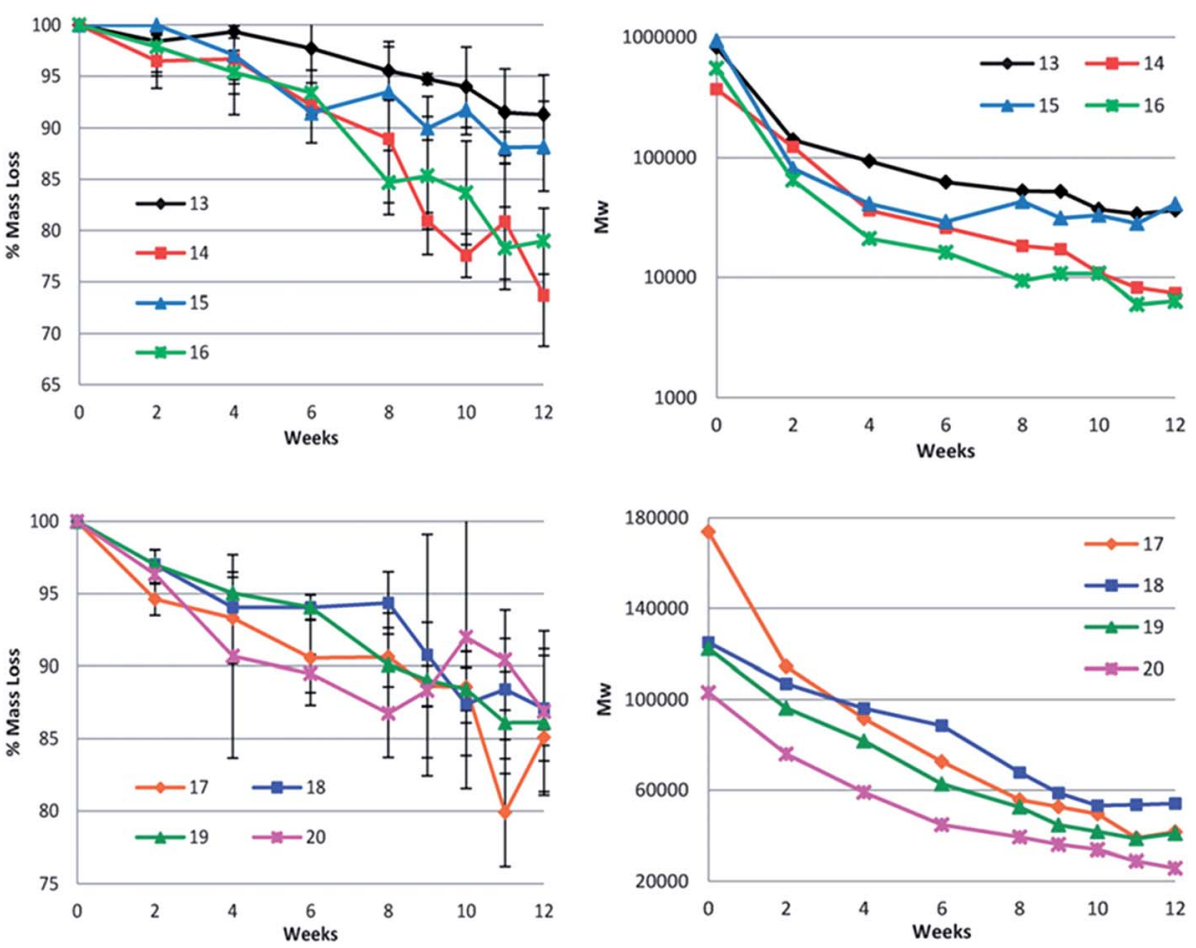

Fig. 2 Percent film mass loss and molecular weight decline of polymers containing alanine and phenylalanine esters. ${ }^{51}$ Copyright 2012 , Royal Society of Chemistry.

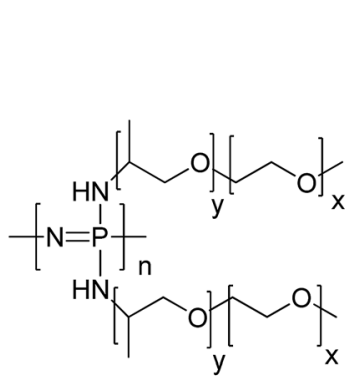

16

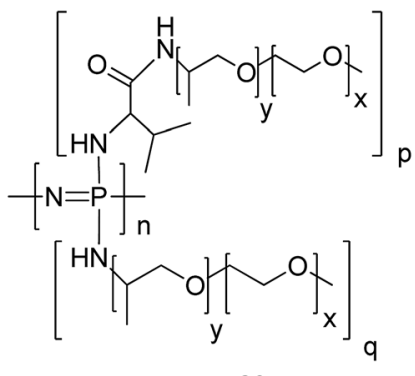

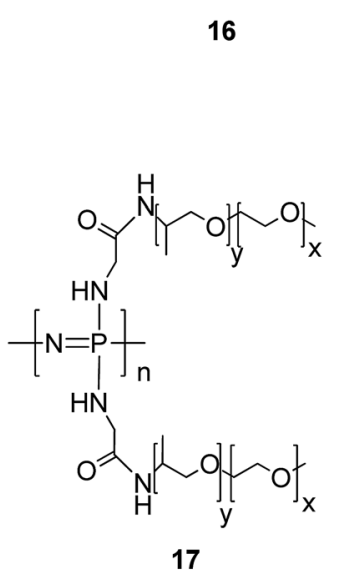

20

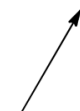

$$
\mathrm{p}
$$

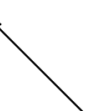<smiles>CN=P(Cl)(Cl)C(C)(C)Cl</smiles><smiles>CI</smiles>

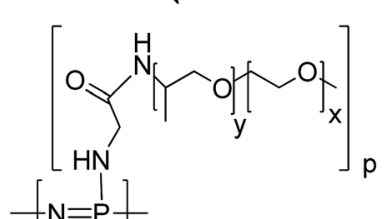


<smiles>[R]NCC(C)(Cl)P(Cl)(Cl)=NC</smiles><smiles>[R]NP(=N)(NC(C)(C)C)N([R])C</smiles>

$\mathrm{R}=$<smiles>CCc1ccc(OC)cc1</smiles>
R

$$
R=
$$<smiles>CCCc1ccc(OC)cc1</smiles>

21

22

Scheme 5 Synthesis of aromatic groups attached through nitrogen. ${ }^{56}$

release the side group and start the degradation process, as shown in Scheme 3. However, some degradation with time may be due to the internal breakdown of side groups, as in the case of amino acid esters (Scheme 4). ${ }^{\mathbf{4 4}}$ Degradation behavior may also depend upon the size of side groups. Smaller side groups with less crowding around the main chain provide more instability to the PPZs as compared to larger side groups of the same kind because they allow the easy attack of water molecules on the bond between phosphorous and the other linking atom. ${ }^{\mathbf{4 5 , 4 6}}$ Molecules with greater crowding may cause greater steric hindrance and reduce the chances of attack. Similarly, the hydrophilic/hydrophobic nature of side groups also play a role in the degradation of PPZs. ${ }^{\mathbf{4 6 , 4 7}}$ Glycine esters, which are more hydrophilic in nature and smaller in size, degrade rapidly than alanine or other amino acid esters. Degradation of some nitrogen-linked PPZs is summarized in Table 1. The other important thing in the selection of side groups is biocompatibility, as they may produce toxic products upon degradation.
Toxic degradation products can mutilate healthy cells, resulting in ineffectiveness of the drug-delivery system.

\subsection{Nitrogen-linked groups}

A number of PPZs have been synthesized in which the same or different side groups have been attached through nitrogen on both sides of main chain, replacing the chlorine atoms. ${ }^{\mathbf{1 0 , 4 9}}$ Mostly, such groups contain amino or imino nitrogen, whose proton is consumable by a base to generate a strong nucleophile. Such nucleophiles attack easily on PDCP to replace chlorine. Some of the substituents may contain more than one functional group. In such cases, other functional groups must be protected before linking the required amino group.

3.1.1 Nitrogen-linked esters. In most of cases of nitrogen linkage, the amino group of amino acid esters is linked with phosphorous. Allcock has reviewed different amino acid, dipeptide and depsipeptide esters that are attached to PPZs through nitrogen. ${ }^{44}$ Jessica et al. have synthesized citronellol esters with glycine, alanine, valine and phenylalanine, and all of these esters were linked with both sides of phosphorous in the polyphosphazene chain, see Table 1 (1-4). PDCP was synthesized by TROP of HCCP under vacuum at $250{ }^{\circ} \mathrm{C}$. All of these polymers are of high molecular weight, with the highest for the glycine ester-containing polymer and the lowest for the phenylalanine-containing polymer. These amino acids, on hydrolysis in deionized water, degraded in similar patterns; i.e. glycine degraded much faster than alanine, valine and phenylalanine citronellol esters. ${ }^{50}$ The presence of a bulkier group adjacent to the point of attachment causes steric hindrance for the attack of water molecules on the main chain, which results in the slowest degradation, as indicated by phenylalanine citronellol esters. As citronellol is an anti-inflammatory agent,

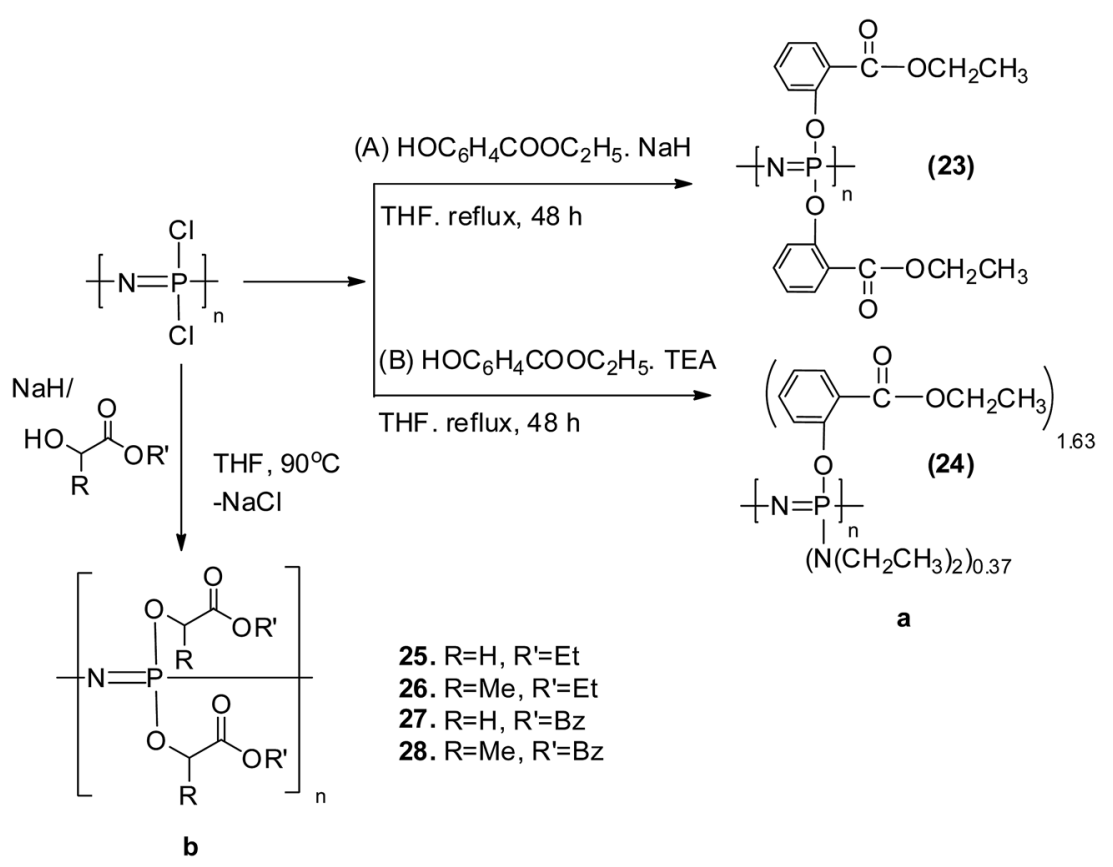

Scheme 6 Synthesis of polyphosphazene containing ethyl esters linked through oxygen: (a) salicylic acid ethyl ester and diethylamine (23-24), ${ }^{27}$ (b) glycolic or lactic acid ethyl and aryl esters (26-28). ${ }^{57}$ 
these polymers can be used for drug delivery. A similar study was done by the same author in which two amino acids, alanine and phenylalanine, were converted into different alkyl esters and linked to the main chain through nitrogen linkage on both sides following the TROP method, see Table $1(\mathbf{5 - 1 2}) .{ }^{\mathbf{5 1}}$ Due to the presence of the bulky group, the increased steric hindrance and decreased mobility of the main chain resulted in higher $T_{\mathrm{g}}$ values for polymers with the phenylalanine alkyl ester as compared to their alanine ester counterparts. Fig. 2 shows the polymer mass loss and molecular weight decline, respectively. According to GPC data of molecular weight decline showing hydrolytic sensitivity, it is clear that the polymers with higher molecular weights underwent the minimum decrease in mass and molecular weights for both types of polymers. Due to their good biodegradability, these polymers can be used for drugdelivery applications. As biomaterials for different biomedical applications share many common properties such as biodegradability and biocompatibility, so materials basically developed for one application can be modified for another. ${ }^{\mathbf{4 6 2}}$

Singh et al., using TROP, synthesized and studied the properties of poly[bis(ethylalanato)phosphazene] and its cosubstituted analogue: poly[(ethylalanato $)_{1}$ (ethylglycinato $)_{1}$ phosphazene] (13-14). ${ }^{46}$ Hydrolytic degradation patterns showed that decreased crowding due to the presence of glycine ester in the latter polymer resulted in greater loss of molecular weight. Another factor may be the initial molecular weight,

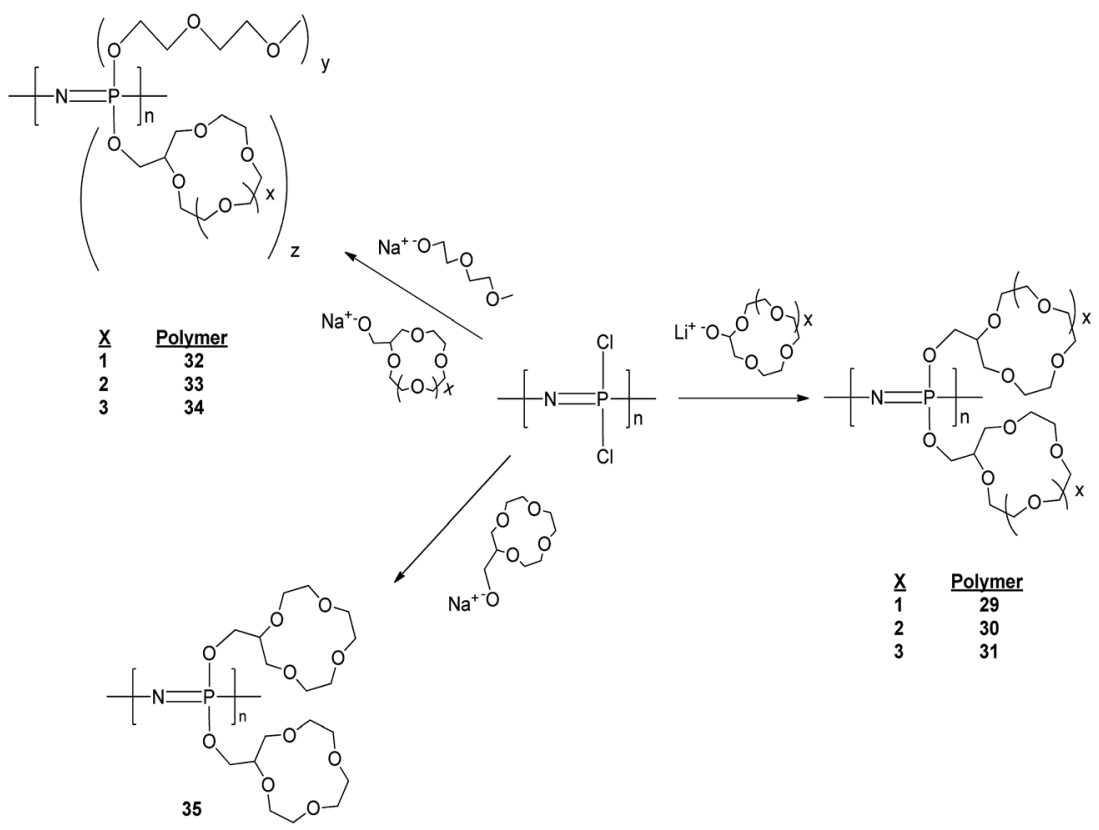

Scheme 7 Synthesis of polyphosphazenes containing oxygen-linked crown ethers (29-35). ${ }^{58,59}$

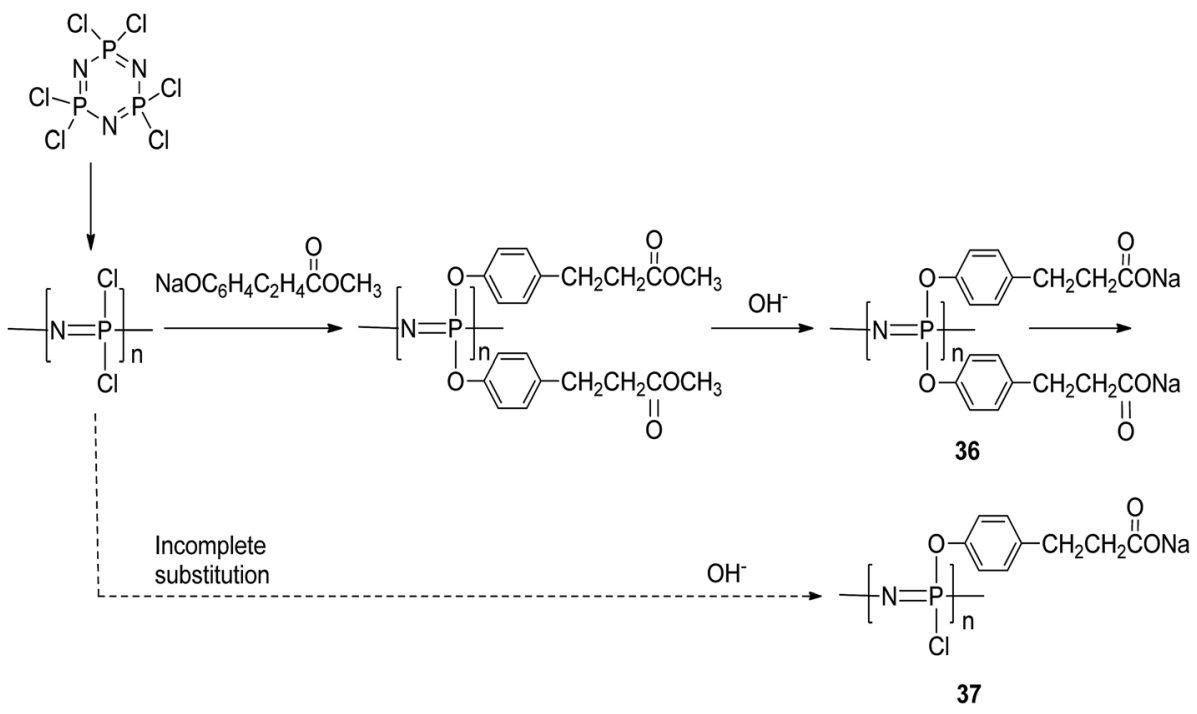

Scheme 8 Synthesis of oxygen-linked aromatic carboxylic acid sodium salt (36-37). ${ }^{60}$ 


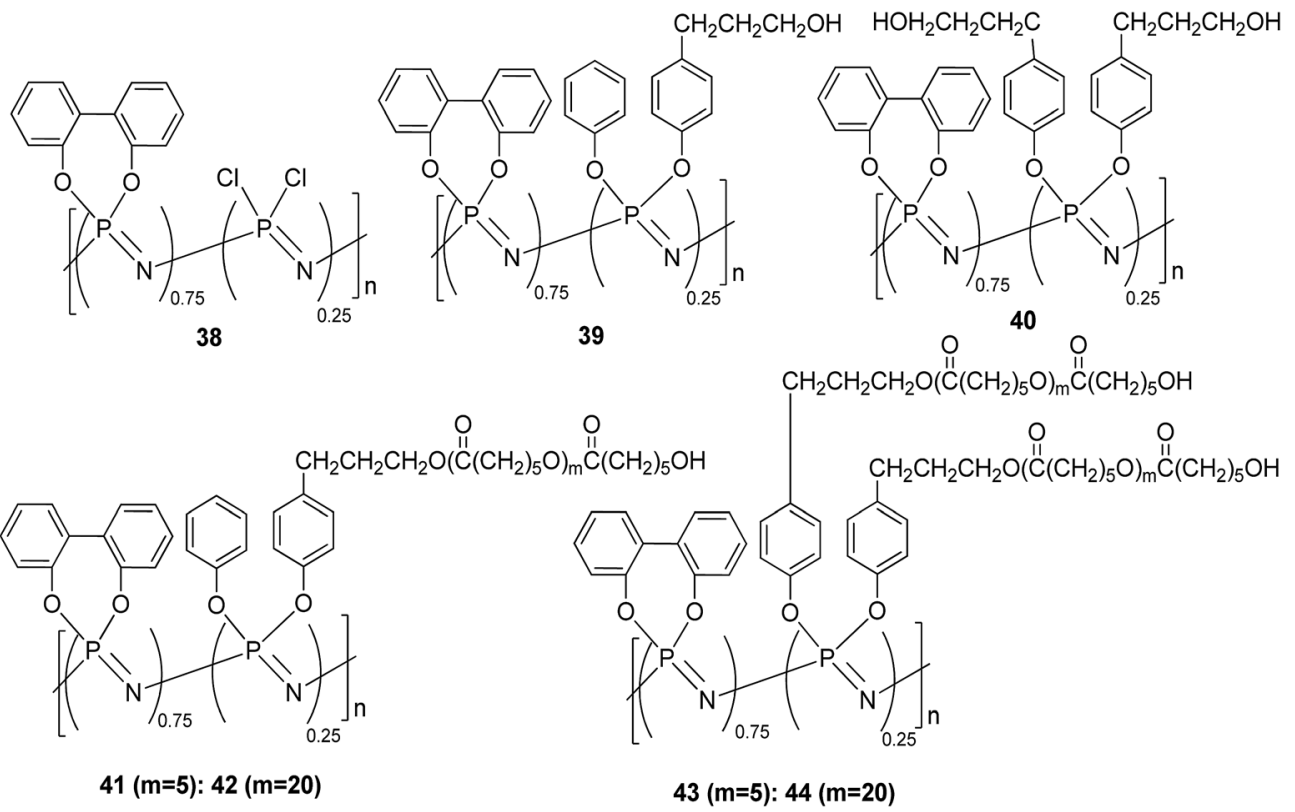

Fig. 4 Oxygen-linked aromatic, polycaprolactone-substituted polyphosphazene. ${ }^{62}$

which is lower and allows more water uptake, resulting in faster degradation. Similarly, PPZs containing ethyl phenylalanato-coethyl glycinato (15), alanine ethyl ester, and phenylalanine ethyl ester-co-imidazole side groups have been prepared using TROP for various medical uses. ${ }^{10,53}$

3.1.2 Nitrogen-linked ethers. Ethers contain oxygen atoms and are mostly hydrophilic in nature. Thus, they are used to increase the hydrophilic nature of the polymers. Polyethylene glycol terminated with amine group is used by many researchers to synthesize water-soluble PPZs. ${ }^{54}$ Wilfert et al. prepared PPZs with a polyethylene glycol linked to the main chain through nitrogen by using LCP of chlorophosphoranimine (Fig. 3).$^{55}$ Five different polymers with or without amino acid spacer showed altered degradation pattern. The slowest degradation was shown by the polymer without any spacer. The glycine spacer resulted in faster degradation compared to the valine spacer, which was due to the greater steric hindrance of the valine around the main chain. All these polymers also showed biocompatibility in cell viability test.

3.1.3 Nitrogen-linked aromatic compounds. Aromatic compounds are mostly hydrophobic in nature, and when such groups are attached to the PPZ main chain, the resulting polymers are mostly insoluble in water. Benzene ring and its derivatives, with greater crowding around the main chain, cause steric hindrance, which may increase their $T_{\mathrm{g}}$ values and decrease hydrolytic sensitivity. 4-Methoxybenzylamine and 4methoxyphenethylamine have been utilized to prepare novel organophosphazenes, i.e., poly[bis(4-methoxy benzylamino) polyphosphazene] (21) and poly[bis(4-methoxyphenethylamino) polyphosphazene] (22) by the replacement of the chlorines of PDCP synthesized by TROP of HCCP (Scheme 5$).{ }^{56}$ These polymers with the aromatic ring showed increased $T_{\mathrm{g}}$ values of $61{ }^{\circ} \mathrm{C}$ and $66{ }^{\circ} \mathrm{C}$, respectively. These polymers were converted into microspheres, which were then loaded with indomethacin and 5-fluorouracil. Comparing the drug loading and drug release

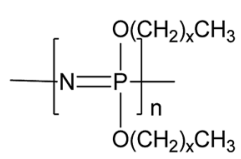

45. $X=1$

46. $X=2$

47. $X=3$

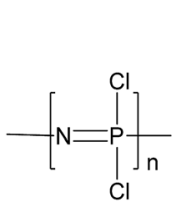

$\mathrm{HOCH}_{2} \mathrm{CF}_{3}$

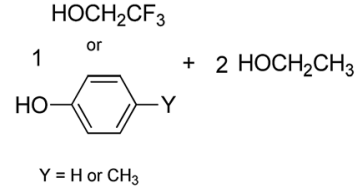

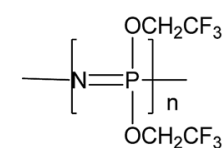

48

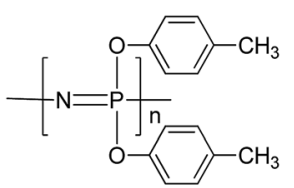

49

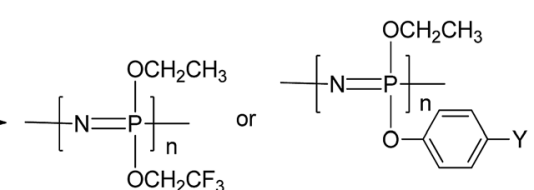

50
51. $Y=H$

52. $Y=\mathrm{CH}_{3}$

Scheme 9 Single-substituent polymers (45-49) and polymers with both ethoxy and O-linked co-substituents (50-52). ${ }^{45}$ 


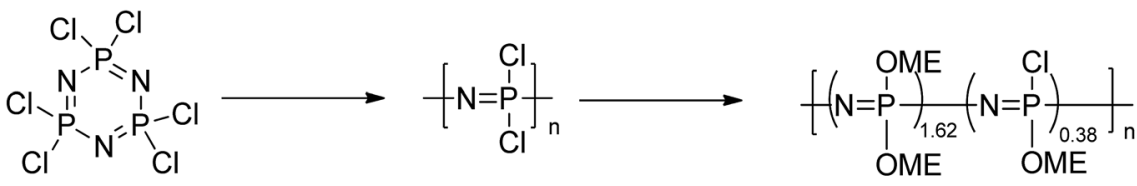

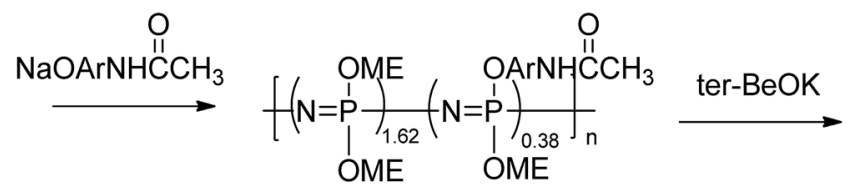

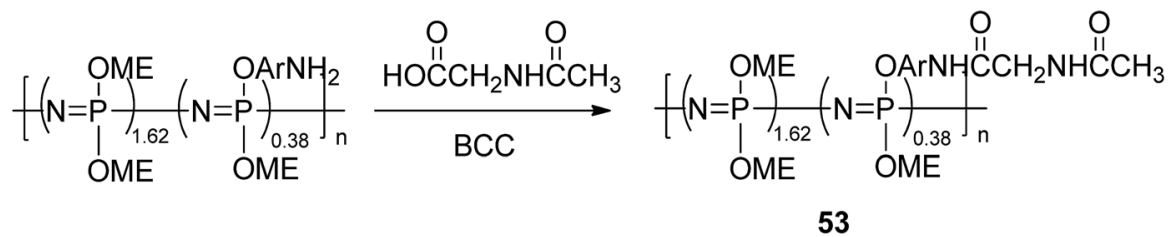

Scheme 10 Synthesis of water-soluble methoxyethoxy-aminoarlyoxy co-substituted polyphosphazenes. ${ }^{63}$

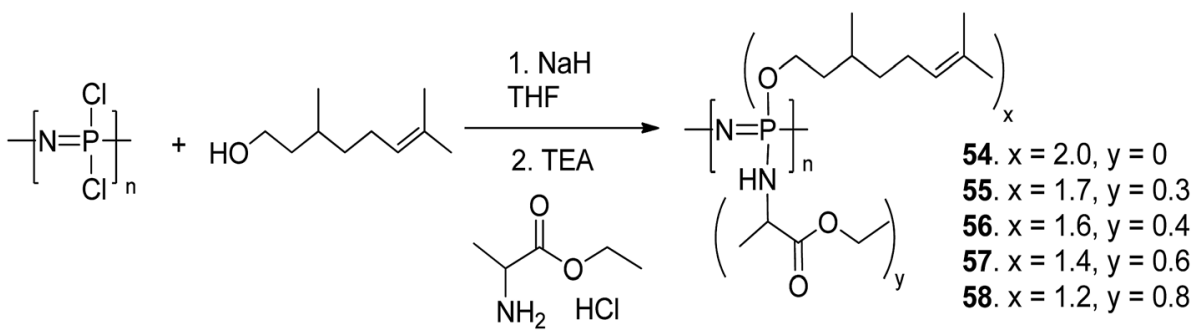

Scheme 11 Synthesis of citronellol-containing polymers. ${ }^{65}$

profile at two different $\mathrm{pH}$ mediums revealed that a greater percentage of loaded drug resulted in faster release of the drug.

\subsection{Oxygen-linked substituents}

The other major linkage of various side groups to the polyphosphazene main chain is through the oxygen atom. Such groups contain one or more hydroxyl groups through which they can be linked to the main chain by releasing protons. Similar to amine-containing groups, a proton-extracting base is used for the attachment of such groups. They can also be further divided into different groups.

3.2.1 Oxygen-linked esters. Poly[bis(ethylsalicylate)phosphazenes] (23), containing ethyl salicylate on both sides of the main chain, and poly[(ethylsalicylate)(diethylamino)phosphazenes] (24), containing ethyl salicylate acid and diethylamine on each side, ${ }^{27}$ have been synthesized by TROP of HCCP. Ethyl salicylate is hydrophobic in nature, while diethylamine is comparatively hydrophilic. The polymer with ethyl salicylate and diethylamine degraded faster than the polymer with both ethyl salicylate groups. Four different poly(organophosphazenes) containing glycolic or lactic acid esters as side groups have also been investigated for their hydrolytic sensitivity (Scheme 6) (25-28).57 Such polymers have much faster degradation rates due to the hydrophilic nature of side groups and increased hydrolytic sensitivity of the polyphosphazene main chain.

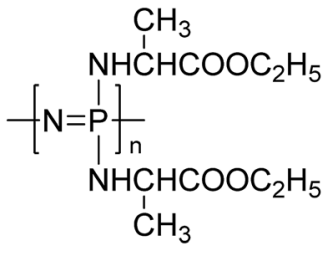

61

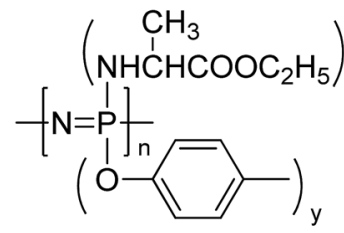

62

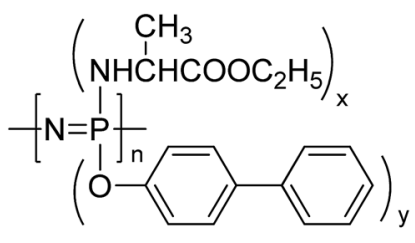

63

Fig. 5 Structures of polyphosphazenes containing alanine ethyl ester in combination with methyl phenoxy and phenyl phenoxy groups (6163). ${ }^{66}$ 

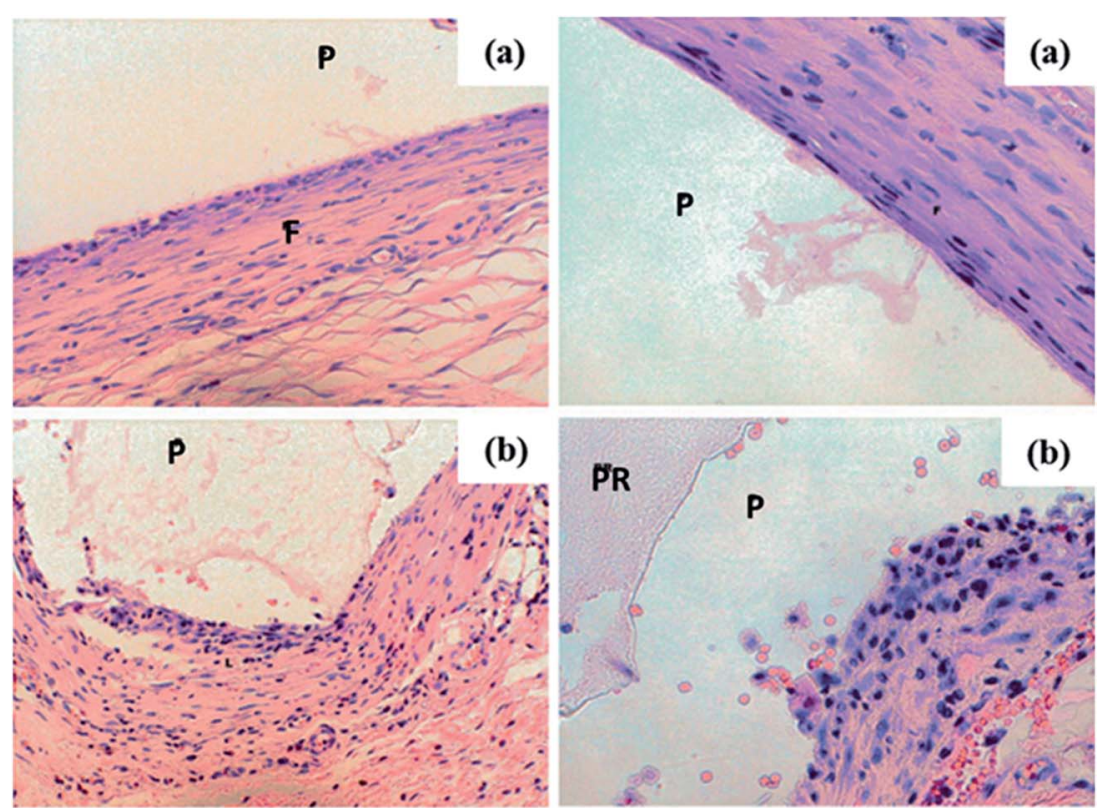

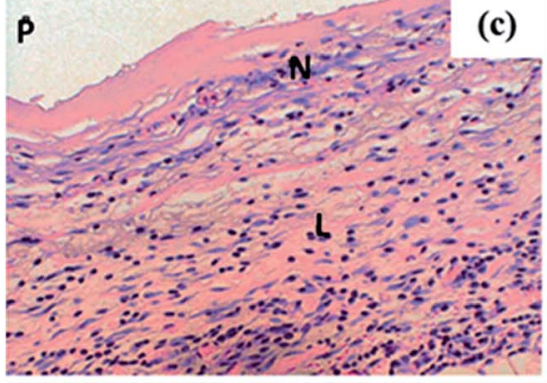

1

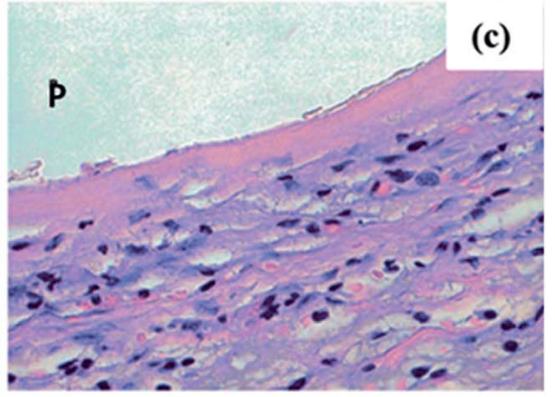

2

Fig. 6 Micrographs of rat subcutaneous tissue response to polymers (a) 61, (b) 62, and (c) 63 after 4 weeks of implantation. P, polymer; N, neutrophils; F, fibrous tissue; PR, polymer residue; $20 \times$ (1) and $40 \times$ (2) magnification. ${ }^{66}$ Copyright 2006 , Wiley Periodicals, Inc.

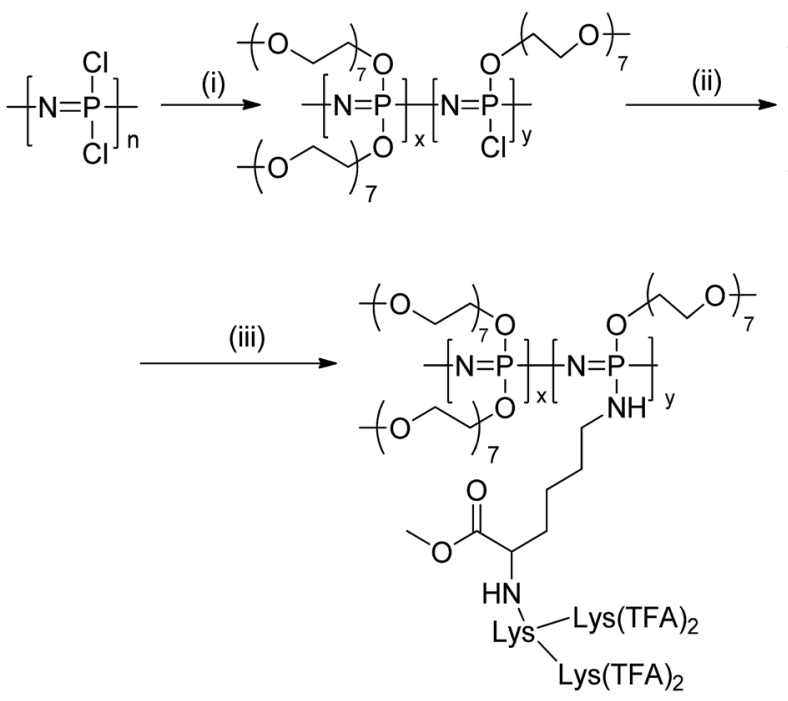

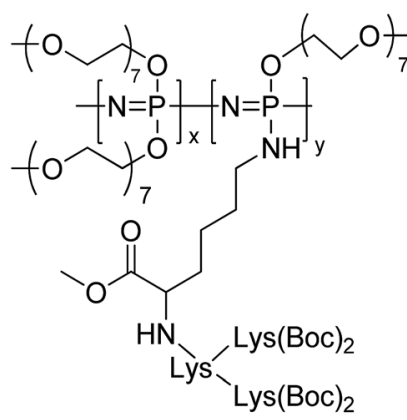

59

60

Scheme 12 Synthetic route to the cationic polyphosphazene bearing MPEG350 and a branched tetra(L-lysine) as side groups (60). Reagents and conditions: (i) $65^{\circ} \mathrm{C}, 8 \mathrm{~h}, \mathrm{MPEG}-\mathrm{Na}$; (ii) tetra(L-lysine) [LysLys $\left.\left(\mathrm{LysBOC}_{2}\right)_{2}\right], \mathrm{NEt}_{3}, 50^{\circ} \mathrm{C}, 2$ days; (iii) TFA. ${ }^{67}$ 

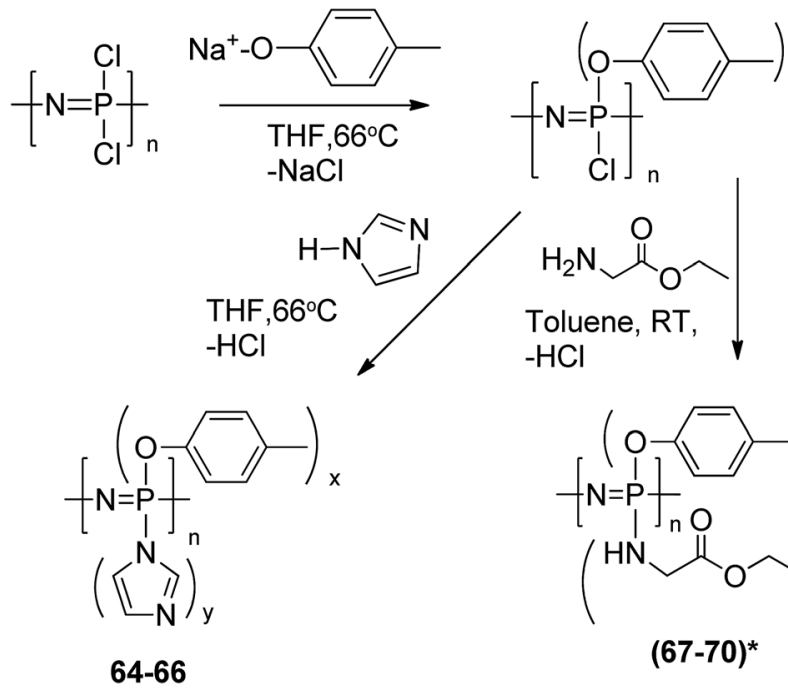
$-\mathrm{HCl}$

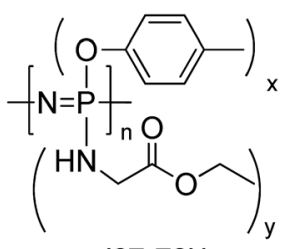

$(67-70)^{*}$

Scheme 13 Synthesis and structures of poly[(imidazolyl)(methylphenoxy)phosphazenes] (64-66) and poly[(ethyl glycinato)(methylphenoxy)phosphazenes] (67-70), ${ }^{7}$ based on the different percentages of side groups.

3.2.2 Oxygen-linked ethers. Oxygen-linked crown ethers have been attached to both sides of phosphorous through oxygen and also in combination with the methoxyethoxy ethanol group on one side, resulting in increased $T_{\mathrm{g}}$ values as compared to poly[bis $\{2-(2-($ methoxyethoxy)ethoxy) $\}$ phosphazene] (29-34). ${ }^{58}$ Cowie and Sadaghianizadeh also synthesized similar crown ethers, such as cantena-poly[bis $(1,4,7,10,13-$ pentaoxacyclohexadecyl-15-oxy)phosphazene] (35) (Scheme
7). ${ }^{59}$ Although their biodegradability and biocompatibility have not yet been determined, this area of research can be explored in the future because ethers provide better solubility to PPZs.

3.2.3 Oxygen-linked aromatic compounds. A large number of aromatic groups have been attached to PPZs, either on one side or on both sides, to attain the required properties. Mostly, aromatic groups impart the hydrophobic character, but there are some modified aromatic groups that can impart hydrophilic nature to polymers. Such organic groups make PPZs hydrolytically degradable so they can be used for drug-delivery applications. Poly[di(sodium carboxylatoethylphenoxy)phosphazene] (36) has been synthesized by TROP (melt polymerization technique) and is a hydrolytically sensitive polymer showing very good vaccine immunoadjuvant potency (Scheme 8). ${ }^{60}$ This polymer was further utilized for intradermal immunization by microfabricated needles by the same author. ${ }^{61} \mathrm{PPZ}$ film containing antigen was fabricated on titanium support to form microneedles, which, when administered to skin, dissolved and released the antigen. The PPZs (39) and (40) are synthesized by the reaction of an intermediate polymer and two hydroxyl groups containing 4-(3-hydroxypropyl)phenol (38) using cesium carbonate as catalyst, which selectively activates the hydroxyl group attached to the benzene ring as compared to the alkylic hydroxyl. ${ }^{62}$ The important thing is the presence of functional alkylic hydroxyl groups used to attach $\varepsilon$-caprolactone, which further undergo ring-opening polymerization in the presence of $\mathrm{Sn}(\mathrm{Oct})_{2}$ (41-44) (Fig. 4). Oxygen-linked ethoxy and fluoroethoxy groups are highly sensitive to water, so bi-substituted products of PDCP synthesized by the TROP of HCCP, having phenoxy or $p$-methylphenoxy on one side and ethoxy group on the other

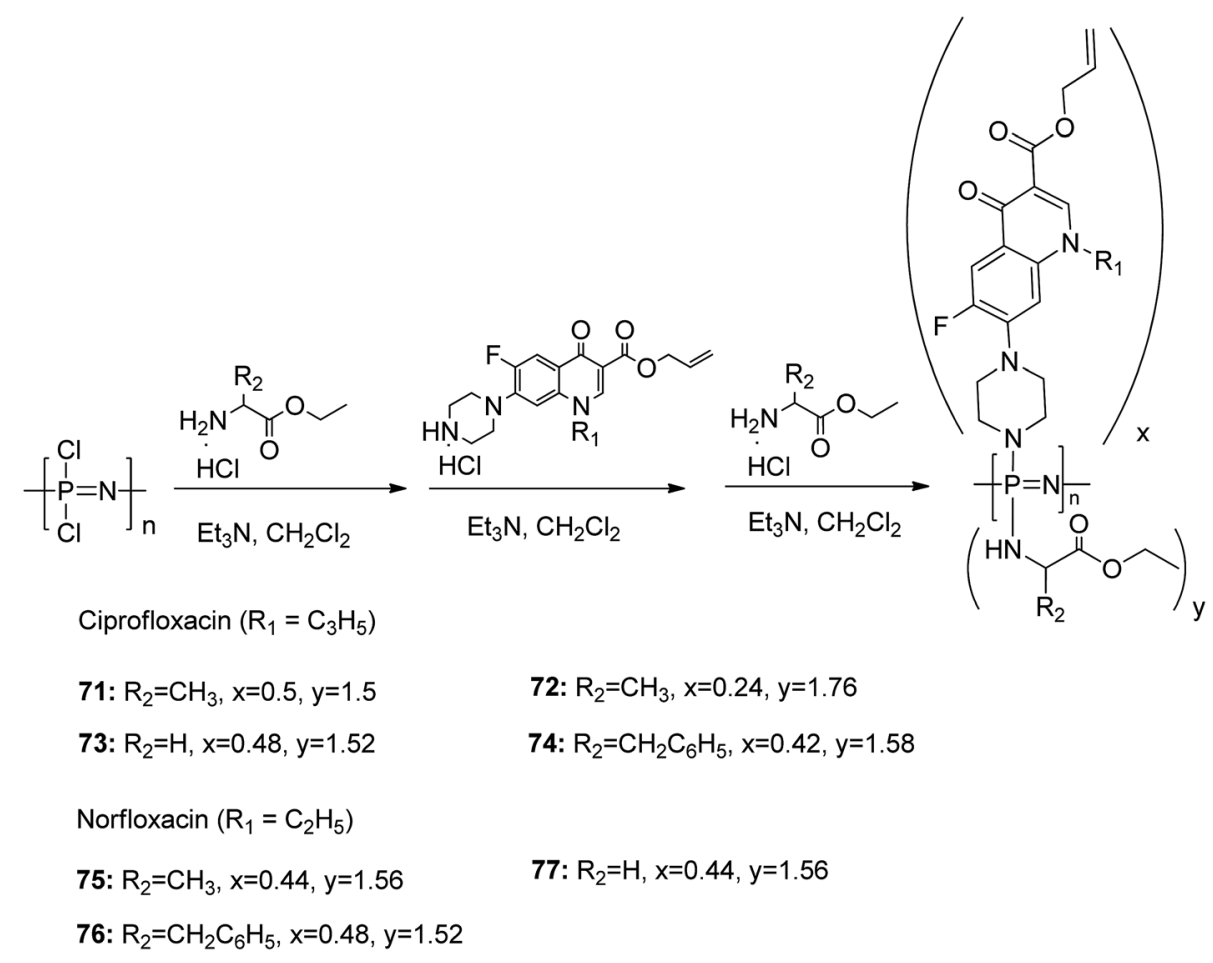

Scheme 14 Macromolecular substitution reaction for the attachment of ciprofloxacin and norfloxacin. ${ }^{73}$ 


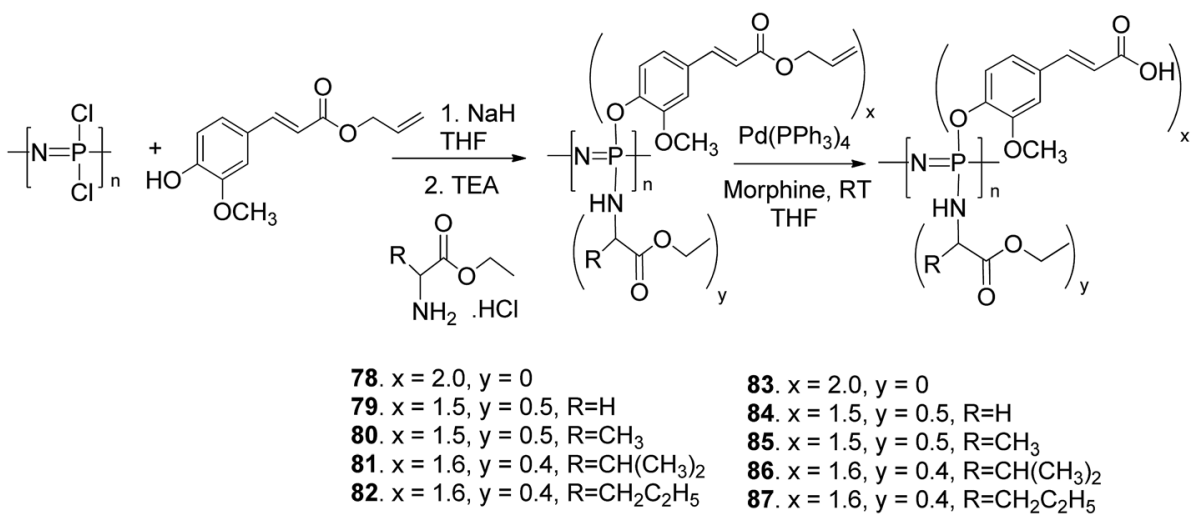

Scheme 15 Synthesis of antioxidant-containing polyphosphazene. ${ }^{76}$

side (Scheme 9) of the main chain, cannot effect its degradability, as shown in a hydrolysis study that continued up to 12 weeks. ${ }^{45}$ Kwon also prepared PPZs for use as carrier of bioactive agents by TROP, with oxygen-linked methoxy on one side and 4acetamidophenol on the other side. The acetamide group was then further modified to produce new derivatives (Scheme 10). ${ }^{63}$

\subsection{Nitrogen- and oxygen-linked groups on the same phosphorous atom}

PPZs show great diversity and flexibility in their synthetic combinations, as many different kinds of aromatic and nonaromatic groups can be linked through both oxygen and nitrogen to the phosphorous at the same time. ${ }^{27,64}$ Citronellol, which has anti-inflammatory and anti-microbial properties, and alanine ethyl ester, which has an amino group, are linked to PDCP (produced by TROP) in different proportions to produce elastomeric PPZs (Scheme 11). Alanine ethyl ester provides hydrolytic sensitivity to the polymers, ranging from 8 to $16 \%$ of mass loss and 28 to $88 \%$ decrease in molecular weight, as checked for 12 weeks. Citronellol can also be cross-linked by the use of UV radiation for different time periods. When crosslinking of citronellol is increased, its modulus is increased, while tensile strength is decreased. ${ }^{65}$

Biocompatibility of mixed aromatic and amino acid esters containing PPZs in rat was determined by Sethuraman and coworkers (Fig. 5). ${ }^{66}$ PPZs with ethylalanato-co-methylphenoxy (62) and ethylalanato-co-phenylphenoxy (63) in $1: 1$ ratio have been synthesized and implanted subcutaneously for 2, 4 and 12 weeks, showing no harmful effects (Fig. 6). PPZ containing methoxy polyethylene glycol as the hydrophilic part and branched tetra L-lysine, LysLys(LysEt) ${ }_{2}$, as cationic moiety were synthesized and shown to interact with DNA (Scheme 12). ${ }^{67}$ These cationic polymers showed zero toxicity and can be utilized for biomedical applications.

Laurencin et al. prepared two different hydrolytically sensitive PPZs that can support the growth of osteoblast cells. These polymers have imidazolyl-co-methylphenoxy and ethylglycinatoco-methylphenoxy side group pairs linked through oxygen and nitrogen atoms (Scheme 13). ${ }^{7}$

\section{Different approaches for drug delivery}

Polyphosphazenes provide a vast field for variation in properties and structural flexibility, so they can be tuned in many ways to be used for drug delivery. They can be modified into copolymers, drug-polymer conjugates, micelles, microspheres, fibers and polymeric blends, which can entrap drugs by different methods and deliver them to the required destination. Here is a brief discussion of these techniques.

\subsection{Polyphosphazene-drug conjugate}

Drug-polymer conjugate is an emerging area of drug delivery in which the drug is chemically linked to the polymer backbone. ${ }^{68}$ In order to combat the hurdles related to the delivery of drugs, such as low solubility, protection against degradation by various factors, low bioavailability and high dose toxicity, drugs are also attached with the polymer chain. ${ }^{69} \mathrm{~A}$ number of such drugpolymer moieties have been synthesized ${ }^{70}$ since its detailed study by Ringsdorf. ${ }^{71}$ Platinum-based anti-cancer drugs have been attached to different polymers, including polyphosphazene, through direct attachments or through different linkers. ${ }^{72}$ Some antibiotics, such as ciprofloxacin and norfloxacin, are directly attached to the polyphosphazene chain in combination with amino acid esters (Scheme 14). The selected polymers are transformed into films and micro/nanofibers, which are hydrolytically sensitive and release the drugs on breakage. But in this case, maximum loading of the drug on the polymer chain is no more than 25 mole percent. According to this study, nano/microfibers are more sensitive to degradation as compared to films. ${ }^{73}$ The release profile of ciprofloxacin has also been studied by linking it to polyurethanes, which are enzymatically degradable. ${ }^{74}$

Hypericin, a naturally occurring biologically active drug with anti-bacterial, anti-viral and anti-tumor activities as well as being a photosensitizer agent, is deficient of good water solubility. Its hydrophilic character was increased by attaching it directly to PDCP along with amine-containing alkylene oxide oligomers. ${ }^{75}$ In another attempt to deliver the anti-oxidant agent ferulic acid, it was attached in combination with the amino acid 


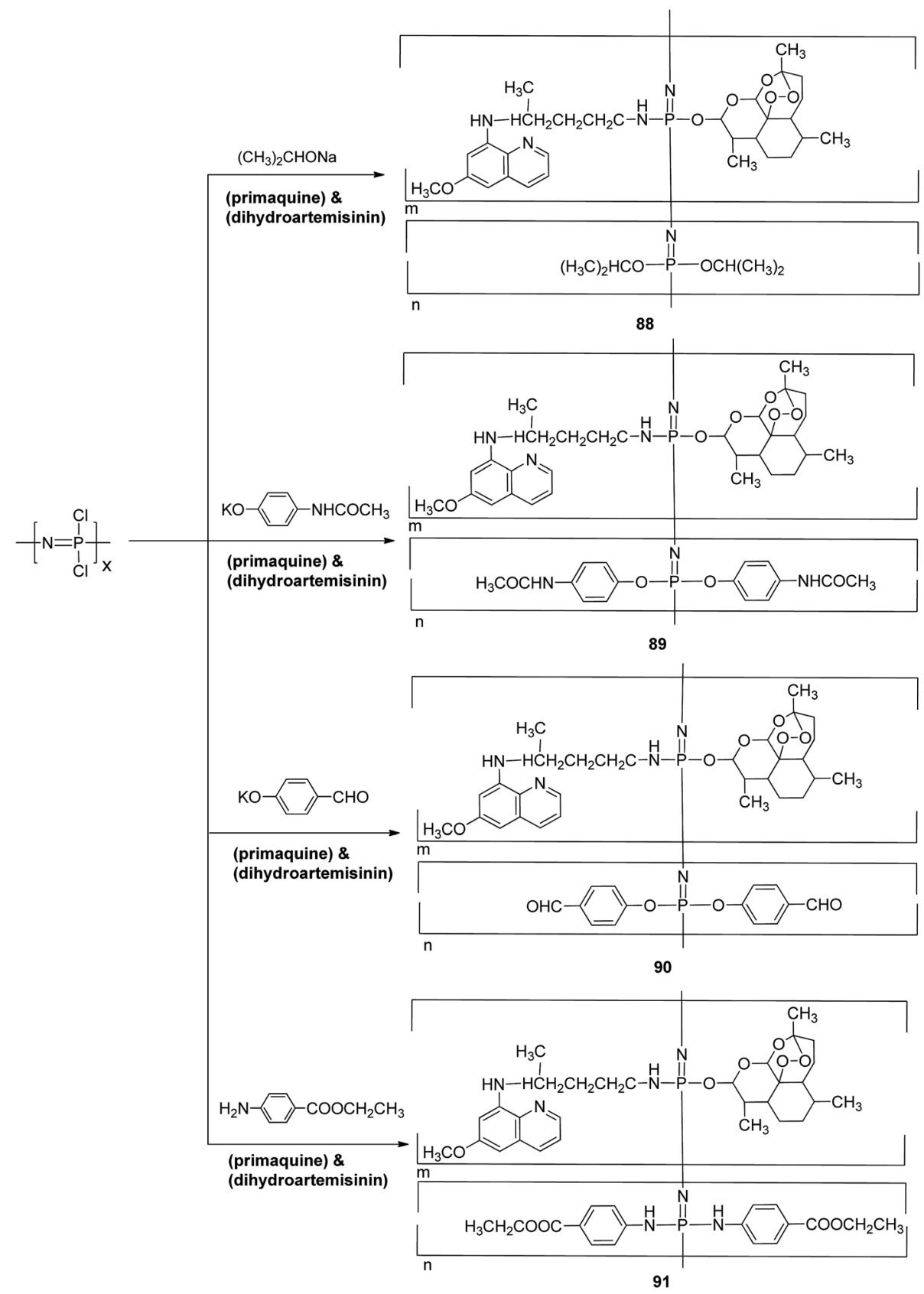

Scheme 16 Synthesis of polyphosphazene-linked, combined conjugates of primaquine and dihydroartemisinin (100-103). ${ }^{77}$

polyphosphazene chain, replacing the chlorines. Ferulic acid was first protected with an allyl ester, treated with sodium hydride and then added to the PDCP solution followed by deprotection. Different combinations of protected ferulic acid (78-82) and unprotected ferulic acid (83-87) along with amino acid esters were prepared, as shown in Scheme 15. The degradation studies showed their hydrolytic sensitivity resulting in 5$25 \%$ decomposition of polymers in an 8 week period. Ferulic acid also showed cycloaddition process under UV radiation, and the crosslinked product was more stable to hydrolytic degradation, resulting in $0-5 \%$ degradation in 8 weeks. $^{76}$
Two anti-malarial drugs, primaquine and dihydroartemisinin, were conjugated at the same time to the polyphosphazene chain in four different combinations of additional substituents (Scheme 16). These conjugates were also formulated into nanoparticles for increased uptake by hepatocytes. The drug release profile showed an initial burst release followed by the sustained release of the drug. These combination drug conjugates also exhibited increased activity and resistance to malaria up to 35 days during in vivo testing of mice. ${ }^{77}$ Allcock and Krause attached adamantane to the main polyphosphazene chain in three different ways. Adamantyloxy, adamantyl amino, and adamantylmethoxy side groups are used in combination 
<smiles>ClP(Cl)(Cl)=NP(N=P(Cl)(Cl)Cl)(N=P(Cl)(Cl)Cl)=P(Cl)(Cl)Cl</smiles><smiles>CCN(CC)N=P(Cl)(Cl)Cl</smiles><smiles>[R]C12CC3CC(C1)C(C3)C2</smiles><smiles></smiles><smiles>[R7]P(Cl)(=NC)C1(C)CC1(C)C</smiles>

$\mathrm{NaOCH}_{2} \mathrm{R}$

$-\mathrm{NaCl}$

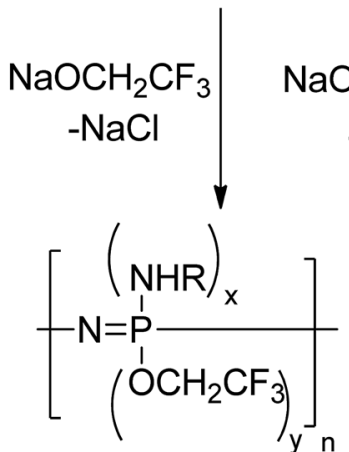

92
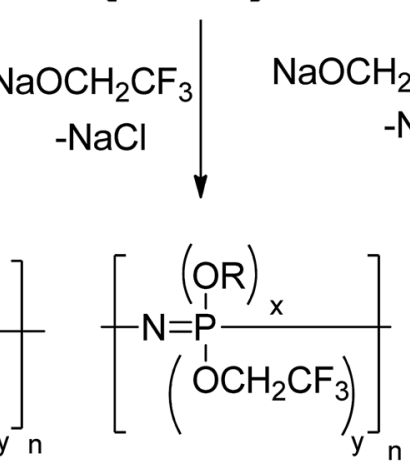

93<smiles>[R]CC([2H])(C)P(Cl)(=NC(C)C)OC</smiles>

Scheme 17 Attachment of adamantyl to polyphosphazene through various linkers. ${ }^{78}$

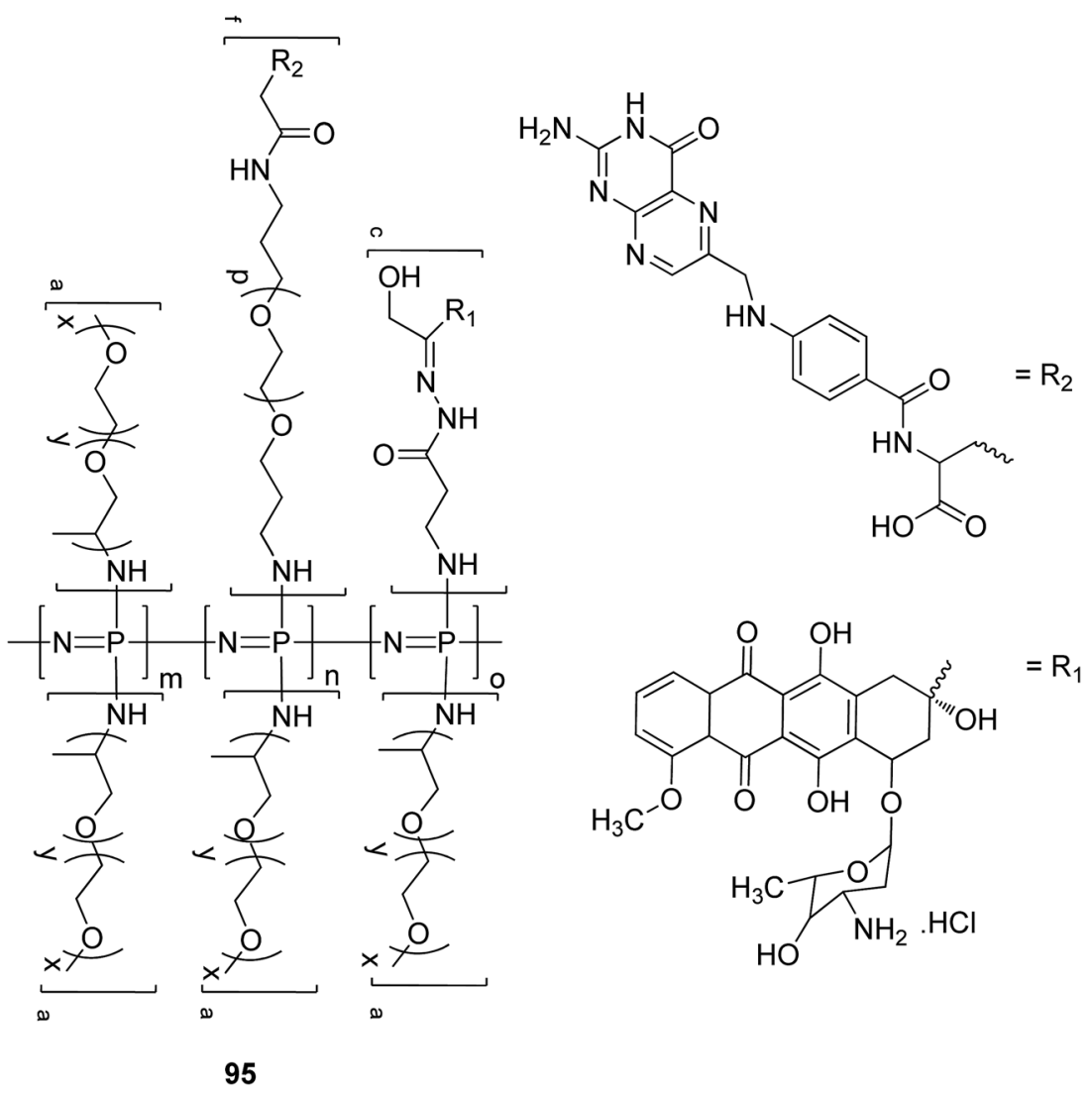

Scheme 18 Epirubicin $\left(R_{1}=\right.$ anti-cancer drug) and folic acid $\left(R_{2}\right)$ attached to the main polyphosphazene backbone. ${ }^{79}$ 


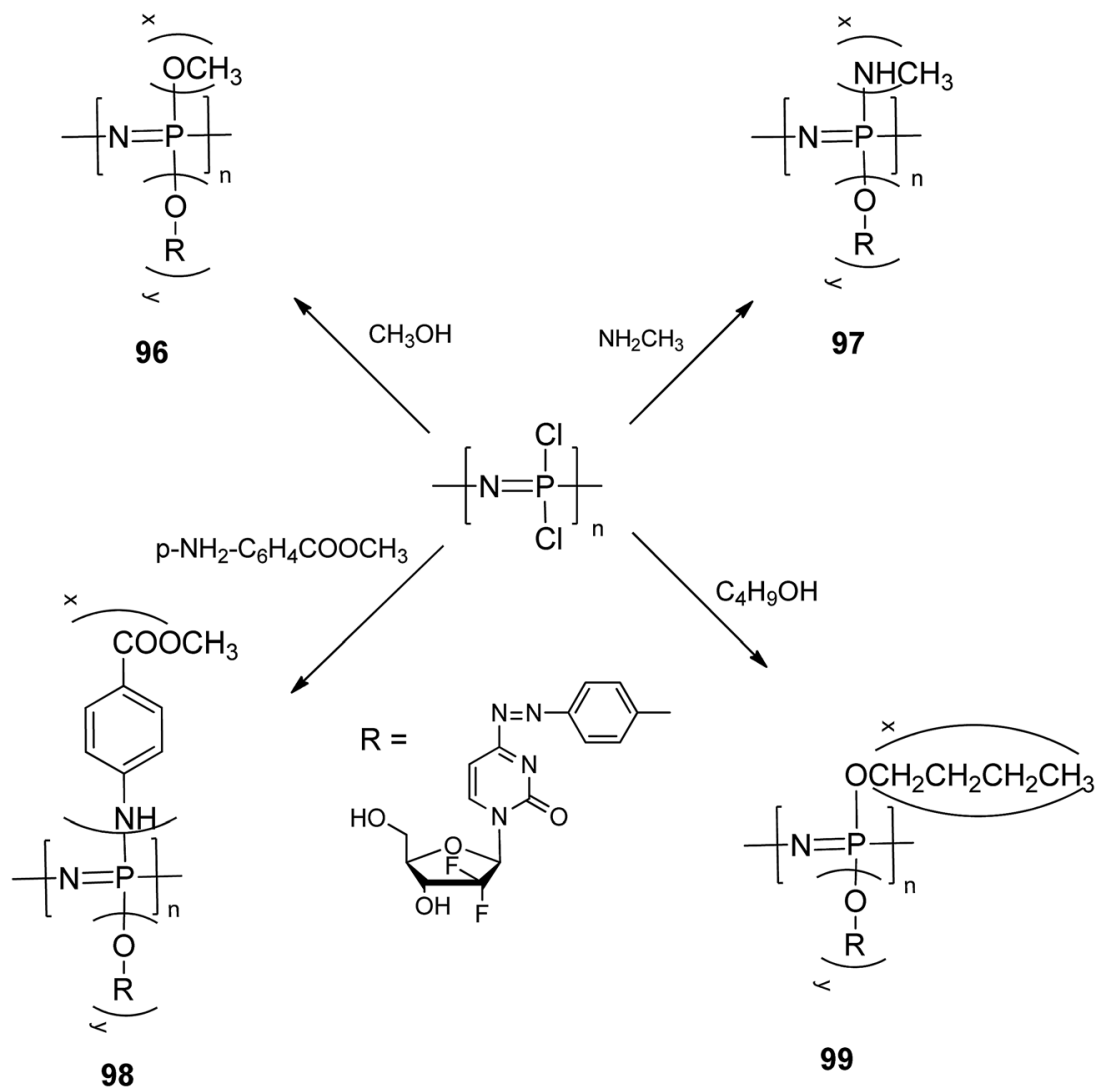

Scheme 19 Synthesis of polyphosphazene azo-based drug conjugates of gemcitabine. ${ }^{80}$

with trifloroethoxy group on the polymer chain as shown in Scheme $17 .^{78}$

Drug-polymer conjugates have also been used to enhance the water solubility of the anti-cancer drug epirubicin, which was attached to the polymer chain of phosphazene along with folic acid through a pH-sensitive hydrazide linker. As the hydrophilic part, an amine-containing polyetheramine copolymer was also attached to the polymer. PDCP was synthesized by ring-opening polymerization from chlorophosphoranimines followed by the addition of linker and copolymer. Drug and folic acid were then attached to the linker chain as shown in Scheme $18 .^{79}$ Similarly, two different anti-cancer drug-polyphospazene conjugates have been synthesized for colon-specific drug delivery, as these conjugates are stable in acidic environment. Methotrexate and gemcitabine, in the form of azo prodrug, were attached to phosphorous in four different combinations (Scheme 19). These conjugates were found to be active against colorectal cancer cell lines. ${ }^{80}$ Recently, Henke et al. introduced polyphosphazene-based anti-cancer prodrug conjugates in which cisplatin and oxliplatin were first converted into prodrugs and then attached to the PPZ chain through a linker. These conjugates released the original drug after intracellular reduction. ${ }^{81}$

\subsection{Polyphosphazene hydrogels}

Hydrogels are the cross-linking network that increase their volume by absorbing a high percentage of water and are being used as carriers in drug delivery. ${ }^{82}$ Chemically, hydrogels can be

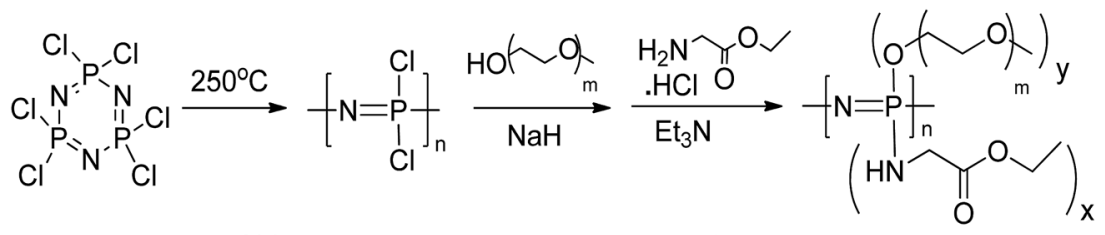

100: $x=0.36, y=1.64, m \sim 6 \quad M_{n}(m P E G)=350$

101: $x=0.44, y=1.56, m \sim 12 \quad M_{n}(m P E G)=550$

102: $x=0.46, y=1.54, m \sim 16 \quad M_{n}(m P E G)=750$

103: $x=0.48, y=1.52, m \sim 44 \quad M_{n}(m P E G)=2000$

104: $x=0.50, y=1.50, m \sim 105 M_{n}(m P E G)=5000$ 

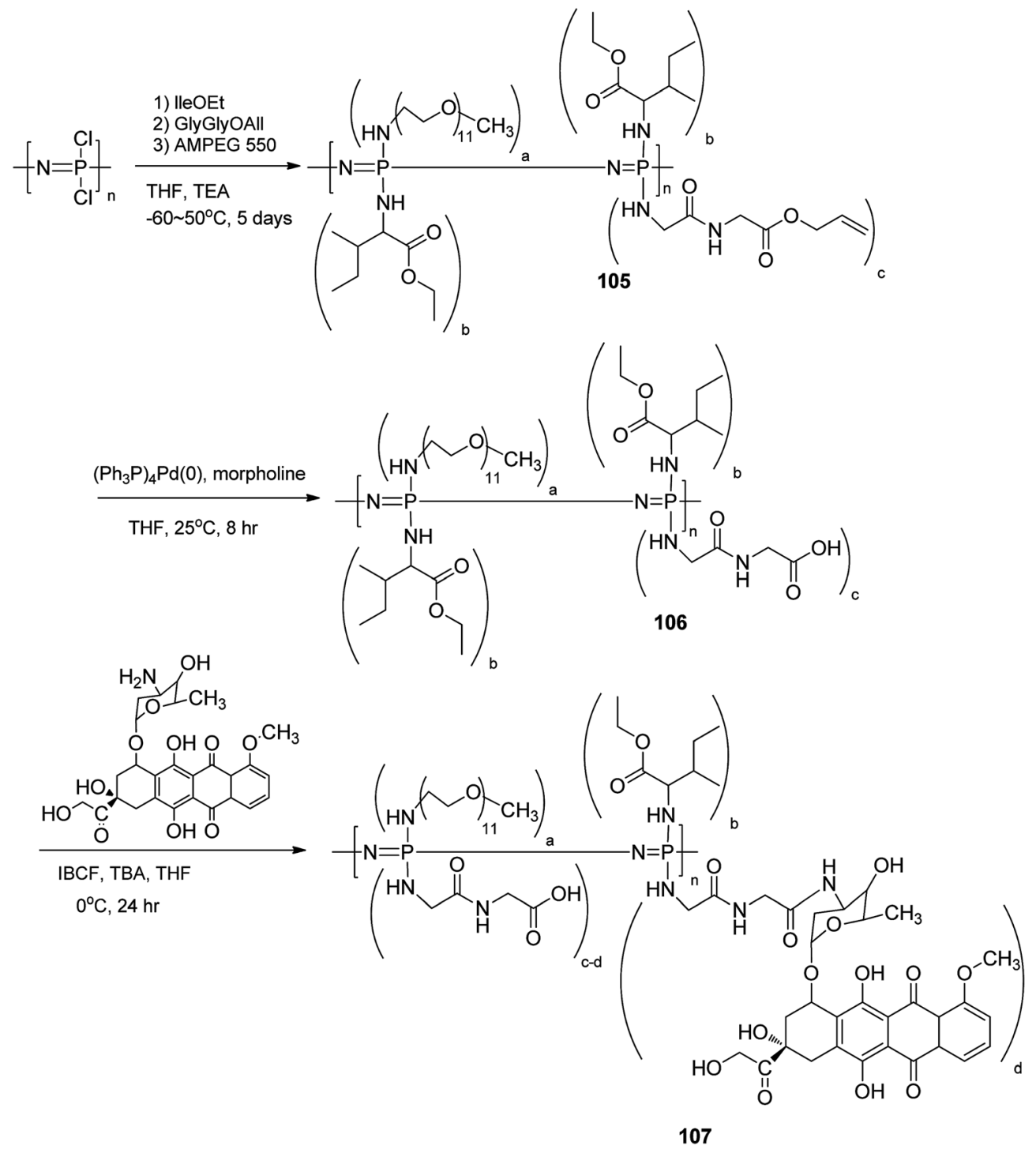

Scheme 21 Procedure for the synthesis of doxorubicin attached to polyphosphazene. ${ }^{54}$

prepared by covalent cross-linking, while physically, they can be produced by some external stimuli such as temperature, UV light or some types of ions. ${ }^{83-85}$ Many thermoresponsive hydrogels $^{86}$ have potential in drug delivery ${ }^{87}$ and tissue engineering, ${ }^{88,89}$ as their degradation products are harmless. ${ }^{90}$ Qian et al. reported the synthesis and application of a new hydrogel based on a methacrylate-substituted polyphosphazene. ${ }^{91}$ Starting from ring-opening polymerization of HCCP and then by nucleophilic substitution, a new poly[bis(methacrylate)phosphazene] has been synthesized that can be used as an injectable
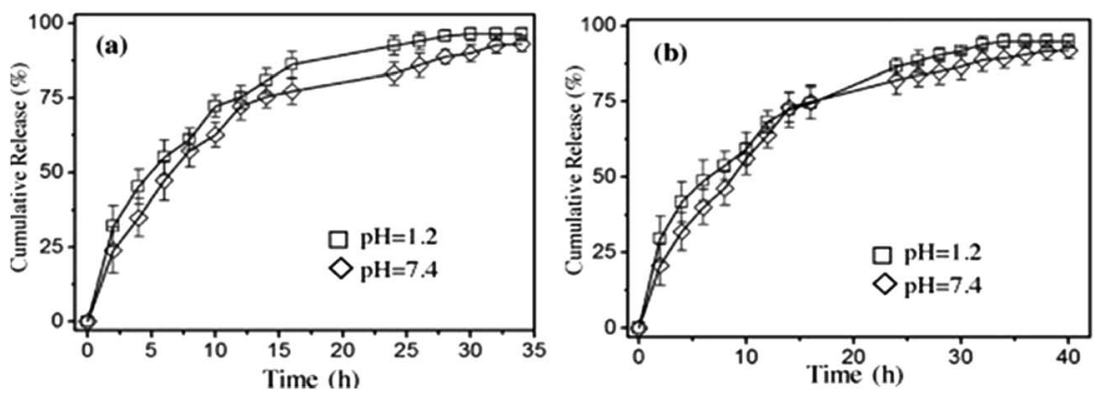

Fig. 7 In vitro model drug-release behavior of (a) vitamin $\mathrm{B}_{12}$ and (b) rhodamine. ${ }^{98}$ Copyright 2014, Elsevier B.V. 

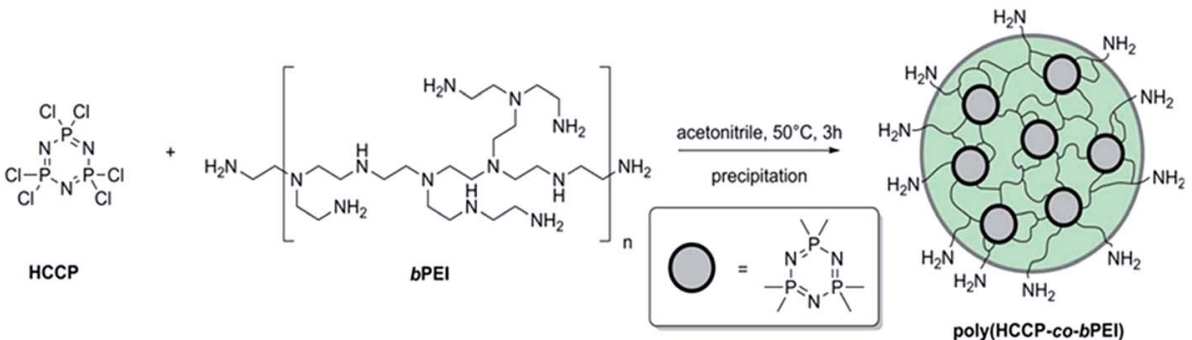

Scheme 22 Synthetic route to poly(HCCP-CO-bPEI) microspheres. ${ }^{99}$ Copyright 2013, Wiley Periodicals, Inc.

(a)

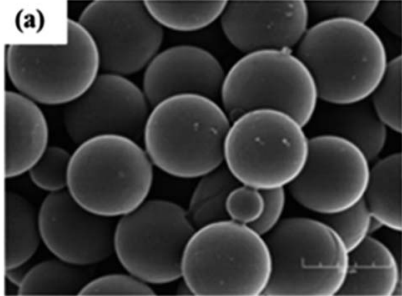

(c)

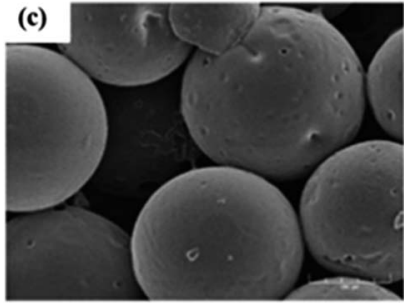

(e)

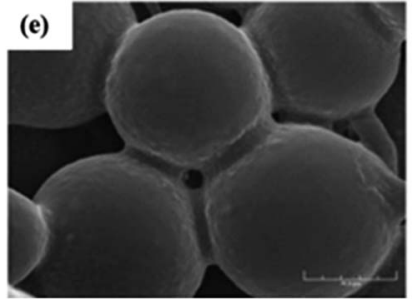

(b)

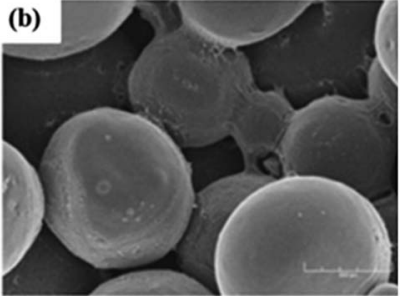

(d)

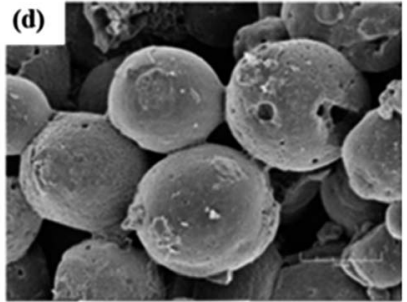

(f)

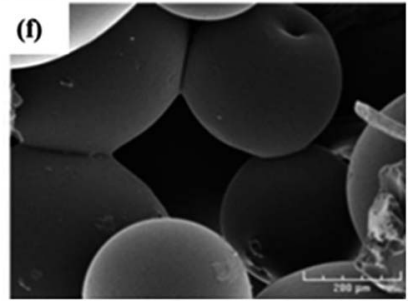

Fig. 8 SEM images of microspheres prepared from PLAGA and different PPZs by solvent/non-solvent method: (a) PLAGA, (b) PNEA, (c) PNEPhA, (d) PNMV, (e) PNEAPhPh, (f) PNEAMPh. ${ }^{100}$ Copyright 2007. Wiley Periodicals, Inc.

hydrogel by host-guest inclusion of biodegradable mixedsubstituted PPZs with the naturally occurring organic molecule $\alpha$-cyclodextrin. Photo-crosslinking was then employed to transform the hydrogel into the solid state with the desired shape. $^{92}$ Similarly, biodegradable poly(organophosphazenes) with different oligo(ethylene glycol) methyl ethers (mPEGs) along with glycine ethyl ester as side chains were synthesized and characterized (Scheme 20). These grafted PPZs and $\alpha$ cyclodextrin were then converted into a supramolecularstructured hydrogel by inclusion complex in aqueous medium. The molecular weight loss of polymers was monitored by GPC to check the biodegradability of hydrogel systems, which revealed that PPZs containing longer chains of MPEG showed better stability and sustained release. ${ }^{93}$

Docetaxel (DTX) is an effective anti-tumor agent with many advantages, but it still faces the problem of low aqueous

solubility. Cho et al. reported the synthesis of injectable and biodegradable poly(organophosphazene) hydrogels that can sustain DTX in aqueous medium. The prepared hydrogels with DTX showed enhanced anti-tumor activity in mice. ${ }^{94}$ The same author also reported an injectable hydrogel system for carrying silibinin, an anti-cancer drug, offering about 2000 times greater solubility. ${ }^{95}$ Another study proved the efficiency of hydrogels as a competent carrier of anti-tumor drug, in which the polymerdrug conjugate of poly(organophosphazene) and doxorubicin (DOX) was synthesized. L-Isoleucine ethyl ester (IleOEt) was attached as hydrophobic group and amino-terminated poly(ethylene glycol) methyl ether (AMPEG 550) as hydrophilic side group (Scheme 21). In vivo studies showed that DOX-containing hydrogels were more effective at inhibiting tumor growth with the least toxicity and could be good carriers of therapeutic agent. $^{54}$ The same author synthesized another paclitaxel-polyphosphazene conjugate-based gel system in which paclitaxel was attached to the main chain through a linker. Hydrolytic degradation, drug release behavior and anti-tumor activity in mice proved it to be more effective than paclitaxel without hydrogel. ${ }^{96}$

\subsection{Polyphosphazene microspheres}

Drug delivery is an advanced approach for the delivery of therapeutic agents at target sites in order to enhance therapeutic efficiency and minimize side effects. Polymeric microspheres are helpful in maintaining the concentration of drugs in a regular manner for a long time, and they can be modified to be injected and target organs, ${ }^{97}$ especially in cancer treatment. Trimethoprim, an antibiotic drug, was directly treated with HCCP as a linker to directly give rise to the microspheres. The amount of cross-linker was reduced by keeping the HCCP constant to achieve microspheres of various sizes. These microspheres are hydrolytically degradable, with enhanced degradation in acidic medium, and can be used for the sustained release of drugs, e.g., rhodamine $6 \mathrm{G}$ and vitamin $\mathrm{B}_{12}$ (Fig. 7).$^{98}$ In another attempt, HCCP was treated with highly branched polyethylenimine (bPEI) in the presence of ultrasonic irradiation using acetonitrile as solvent (Scheme 22). By precipitation polymerization, cyclomatrix cross-linked polyphosphazene was converted into microspheres with high thermal stability. Interesting in these microspheres was the presence of active amine groups that can be utilized for various applications. ${ }^{99}$ Although the authors have not revealed its drug 

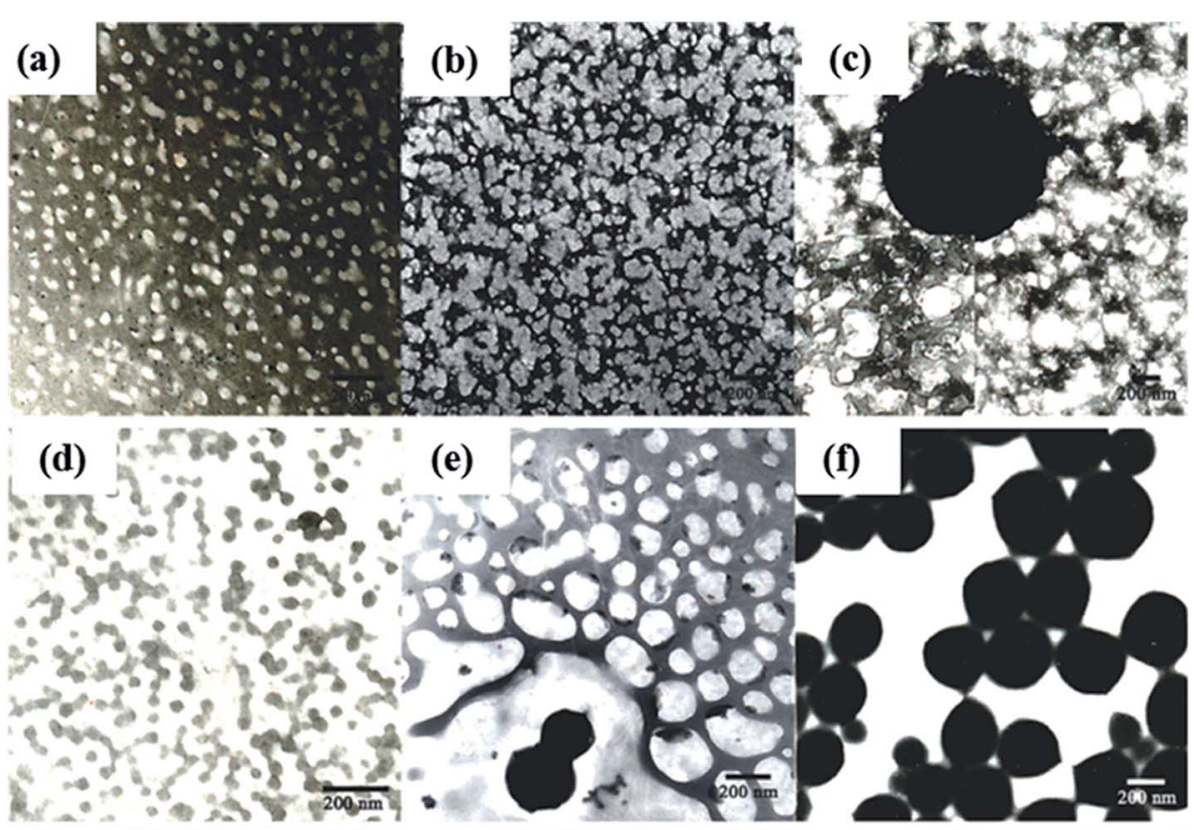

(e)
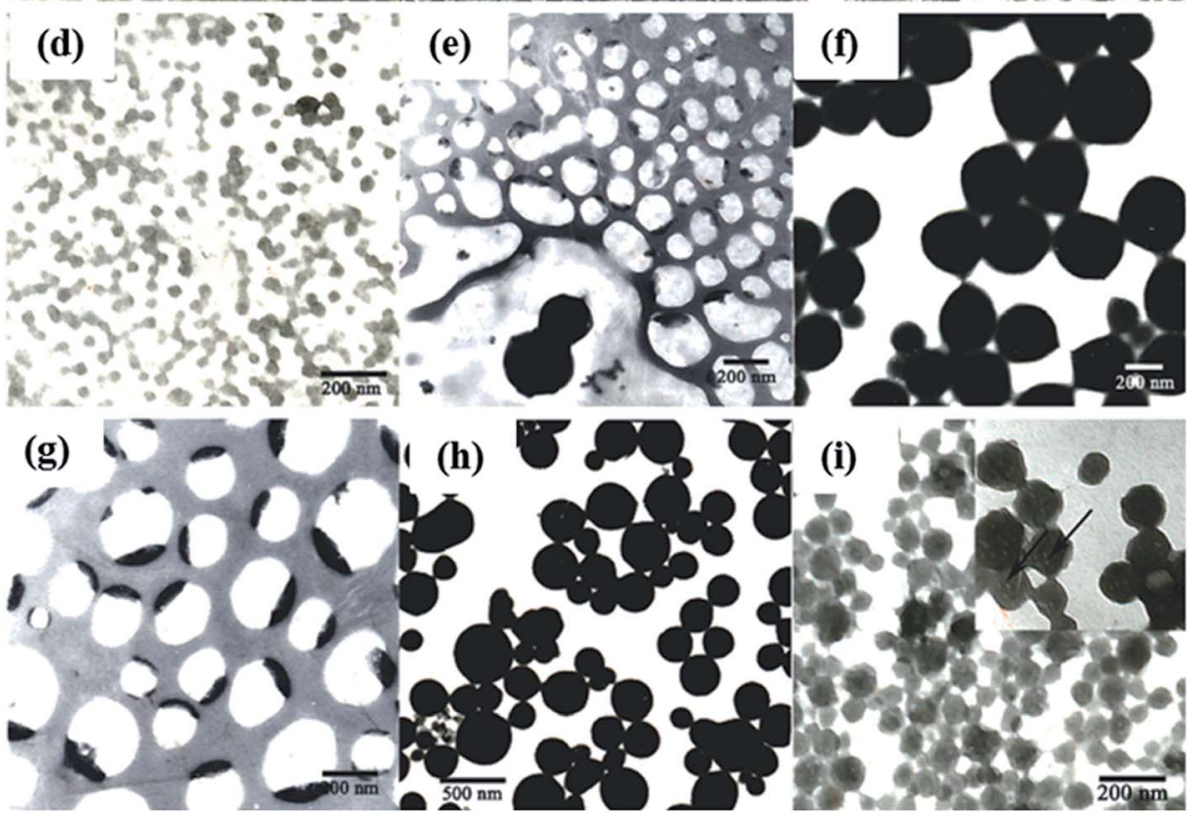

Fig. 9 TEM images of aggregates made from amphiphilic copolymers: (a) copolymer 1, $5 \mathrm{mg} \mathrm{mL}^{-1}$; (b) copolymer 1, 2 mg mL ${ }^{-1}$; (c) copolymer 2, $5 \mathrm{mg} \mathrm{mL}^{-1}$; (d) copolymer 3, DMF as solvent; (e) copolymer 3, DMAc as solvent; (f) copolymer 3, DMAc as solvent, cutoff aggregates after being filtered through a $0.22 \mu \mathrm{m}$ microporous membrane; (g) copolymer 3, DMAc as solvent, filtrate after being filtered through a $0.22 \mu \mathrm{m}$ microporous membrane; (h) copolymer 3, DMSO as solvent; (i) copolymer 3, THF as solvent. ${ }^{115}$ Copyright 2006, American Chemical Society.

release applications, it could probably be important in drug delivery due to the presence of amino groups, which can attach to drugs by $\mathrm{H}$-bonding. Microspheres with controlled porosity and average pore diameter and with better mechanical properties have also been fabricated by using the solvent/nonsolvent method. Five different PPZs and PLAGA are used for microsphere formation (Fig. 8) with a wide range of glass transition temperatures under normal conditions, making it a suitable method of fabricating microspheres for drug-delivery applications. ${ }^{100}$ Efforts are being done to overcome the problems of initial burst release ${ }^{\mathbf{1 0 1}}$ and lack of proper drug release kinetics ${ }^{102}$ for the polymeric microspheres. Caliceti et al. prepared microspheres based on PPZs and demonstrated the release profile of insulin. ${ }^{103}$

Similarly, poly[di-(carboxylatophenoxy)phosphazene] and sulphonated polyphosphazene, which upon the addition of spermine resulted in the formation of microspheres, were used for the encapsulation and release of proteins. ${ }^{104}$ Sustained release of hydrophobic indomethacin and hydrophilic 5-fluorouracil by synthesized PPZs microspheres have also been

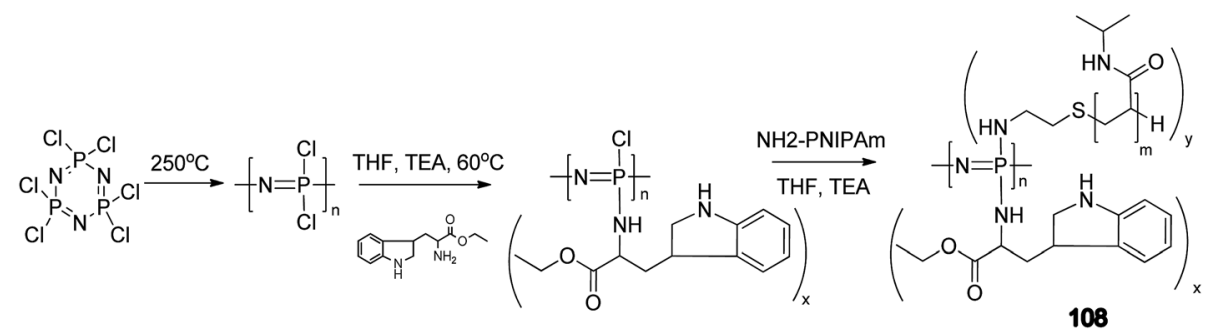

Scheme 23 Synthesis of amphiphilic graft phosphazenes with EtTrp and oligo-PNIPAm as side groups. ${ }^{116}$ 


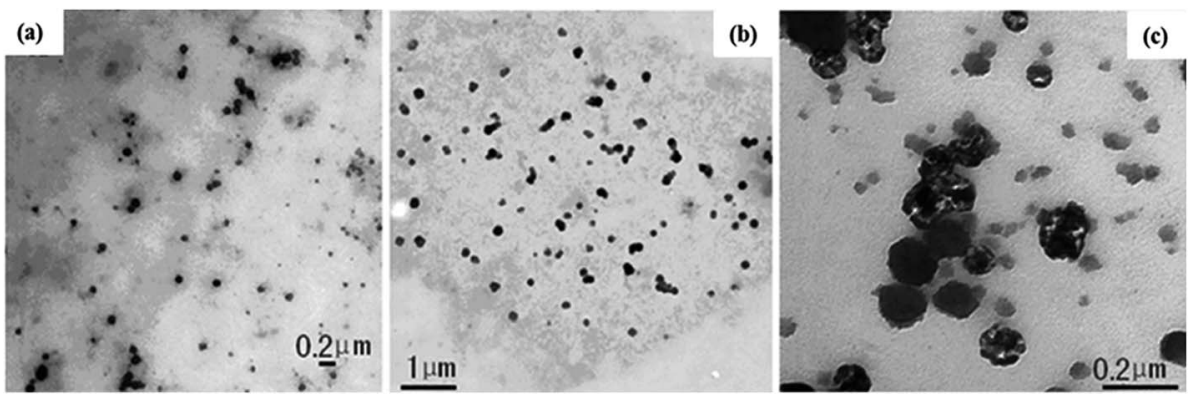

Fig. 10 TEM micrograph of DOX loaded micelles: (a) DOX-loaded micelles based on PNDGP-2 prepared by emulsion method with LC of 20.7\%; (b) DOX-loaded micelles based on PNDGP-3 prepared by emulsion method with LC of $20.7 \%$, at $20000 \times$ magnification; (c) the same as B but at $120000 \times$ magnification. ${ }^{128}$ Copyright 2008, Springer Science + Business Media, LLC.

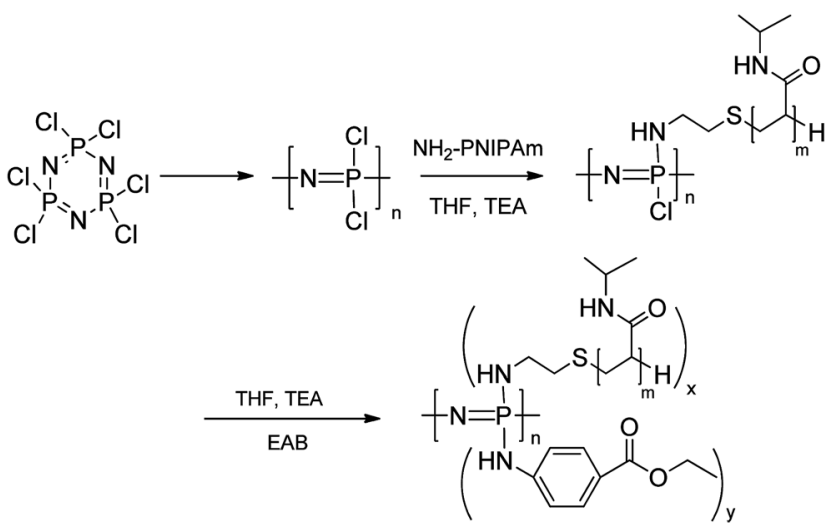

109
Scheme 24 Synthesis of amphiphilic polyphosphazene (PNIPAm/ EAB-PPP) bearing both oligomer PNIPAm and EBA as side groups via macromolecular substitution. ${ }^{137}$

studied. ${ }^{56}$ Microspheres have been prepared by the crosslinking of polyphosphazene hydrogel, in which calcium ions serve as connector. These microspheres are suitable for use in the delivery of proteins, especially vaccines. Poly[di(carboxylatophenoxy)phosphazene], in the presence of $\mathrm{NaCl}$, produced coacervate microdroplets, which cross-linked by the addition of calcium ions to produce microspheres. The technique is considered to enhance the microencapsulation of proteins with high reproducibility and narrow size distribution. ${ }^{105}$ Polyphosphazene containing phenylalanine ethyl ester along with imidazole as side groups was converted into microspheres by three different methods. Different methods had different effects on size distribution of the microspheres. These microspheres were used for the loading and release of naproxen as model drug. ${ }^{106}$

\subsection{Copolymeric micelles of polyphosphazene}

PPZs have huge capacity to accommodate a number of organic/ inorganic groups as well as other polymers to form different kinds of graft and block copolymers, ${ }^{\mathbf{1 0 7 , 1 0 8}}$ which are gaining much attention. The self-assembly of amphiphilic copolymers resulted in a variety of structures, including vesicles, spherical micelles, rod-like micelles, and lamellae. ${ }^{109,110}$ Copolymeric selfassemblies offer the advantages of increased stability and toughness, ${ }^{111}$ as well as various possibilities to modify physicochemical and biological properties by changing the block length, chemical structure and interactions with biomolecules. These properties make the copolymer highly interesting for numerous applications such as transfection vectors, ${ }^{112}$ protective shells for sensitive enzymes ${ }^{113}$ and drug-delivery systems. ${ }^{114}$ Zhang et al. prepared different copolymers with different ratios of $N$-isopropylacrylamide and ethyl-4-aminobenzoate attached to the main chain of PPZs and monitored their different selfassembled morphologies in different solvents (Fig. 9). ${ }^{115}$ These can be used for different biomedical applications, including drug delivery.

The same author prepared amphiphilic graft copolymer based on PPZs. In this study, tryptophan ethyl ester (EtTrp) and poly( $N$-isopropylacrylamide) (PNIPAm) were used as side groups of the main chain (Scheme 23). ${ }^{116}$ Morphology of the selfassembled particles at different conditions were presented in this study.

Using high catalyst concentration (10 weight\%) in the thermal ring-opening polymerization of HCCP, low molecular weight poly(4-methylphenoxyphosphazene)-graft-poly(2-methyl2-oxazoline) was obtained. Bromination of side group 4-methylphenoxy was done, which was further used for the polymerization of 2-methyl-2-oxazolineto to produce graft copolymer. Micelles produced by self-assembly in water entrapped the hydrophobic and fluorescent pyrene as a model drug. ${ }^{117}$ In another study, to increase the efficiency of proton exchange, membranes for block polystyrene-grafted PPZs were developed that contained alkylsulfonic acids as substituents. ${ }^{118,119}$

Copolymeric micelles consist of a hydrophobic core surrounded by a hydrophilic shell, which are usually prepared by dialysis technique. Hydrophobic drugs can be easily captured by the core during the self-assembly of micelles in dialysis bags where solvent is exchanged by water in most cases. ${ }^{120,121}$ Polymeric micelles proved to be excellent vehicles for the delivery of hydrophobic drugs, especially in the case of anti-cancer drugs. ${ }^{122-124}$ There are also $\mathrm{pH}$-responsive and redox-responsive micelles based on different kinds of polymers. ${ }^{125-127}$ Amphiphilic graft polymer 


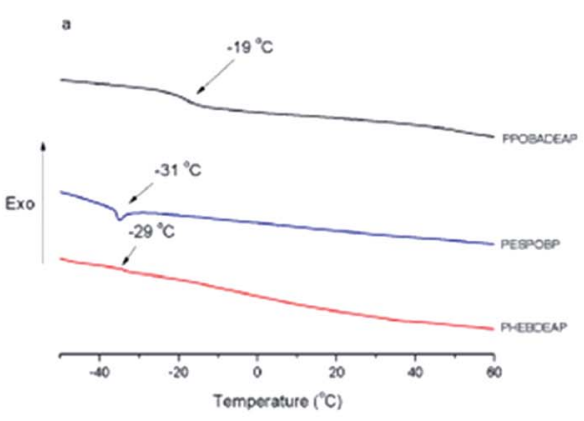

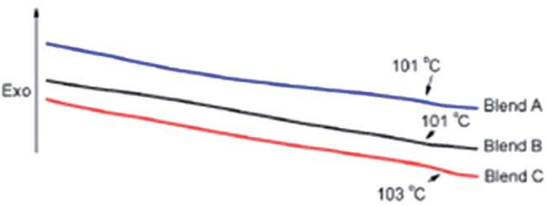
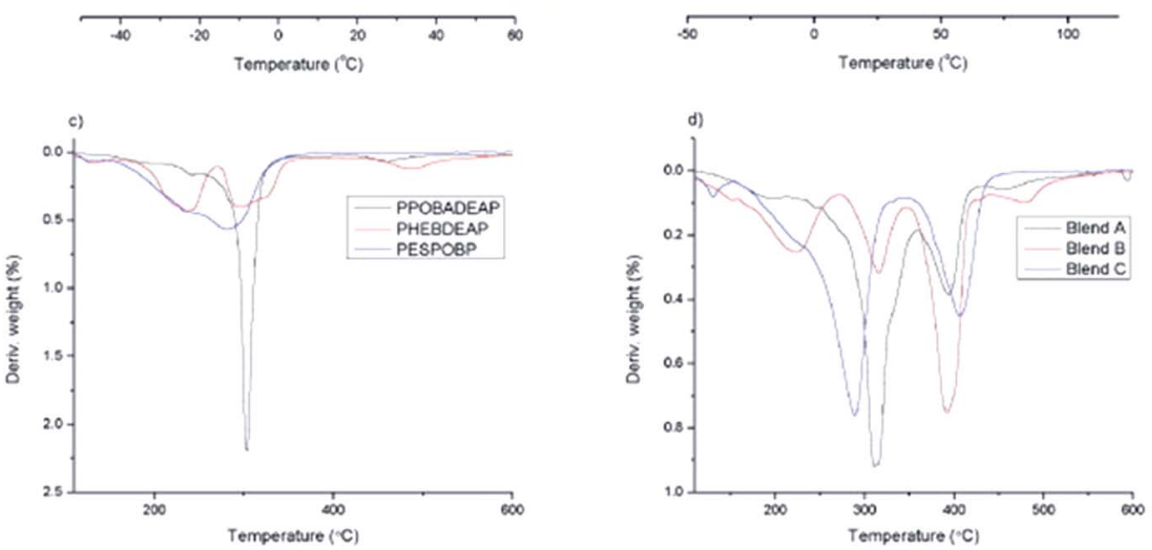

Fig. 11 DSC curves of (a) PPOBADEAP, PHEBDEAP and PESPOBP, and (b) blends A, B and C; (c) DTG analysis of pure PPHOSs; (d) DTG analysis of blends. ${ }^{140}$ Copyright 2015, Elsevier B.V.

based on PPZ containing hydrophobic and hydrophilic side groups can be used for the preparation of micelles and loading of drugs. Doxorubicin was loaded by dialysis method in the micelles produced (Fig. 10) by graft polymers consisting of different ratios of poly( $N$-isopropylacrylamide-co- $N, N$-dimethylacrylamide) (PNIPAmDMAA) and ethyl glycinate around the main chain of polyphosphazene. ${ }^{128}$ Synthesized micelles were temperature sensitive and released encapsulated material as the temperature changed. Thermal-responsive behavior of micelles based on PNIPAmDMAAethyl glycinate PPZ was explored by turbidity method. Lower critical solution temperature (LCST) was different in water and PBS solution.

Diflunisal, an anti-inflammatory drug, was also loaded in temperature-sensitive PPZ-based micelles. At lower temperature, micelles showed burst release, while at body temperature, they showed sustained and controlled release. ${ }^{129,130}$ Zheng et al. also loaded doxorubicin in newly synthesized graft polyphosphazene micelles containing methoxy-poly(ethylene glycol) and glycine ester components with different morphologies. Cytotoxic assay showed the efficiency of these micelles in human breast cancer cells. ${ }^{131}$ In another study, similar amphiphilic graft copolymers based on PPZ were synthesized consisting of MPEG and tryptophan ethyl ester in different ratios. Micellization behavior showed that the critical micelle concentration of copolymers decreased with increased hydrophobic content. DOX was again loaded as an anti-cancer drug against HeLa cells and showed comparable cytotoxicity as compared to free DOX. ${ }^{132}$ Rheumatoid arthritis is a disease related to swelling, stiffness and joint pain, ${ }^{133,134}$ which is treated by various ways including intra-articular and oral dosage forms. ${ }^{135}$ Indomethacin-loaded micelles were introduced in a rat for the treatment of arthritis. ${ }^{136}$ These micelles loaded with indomethacin were prepared by amphiphilic polyphosphazene copolymers with poly( $N$-isopropylacrylamide) and ethyl 4-aminobenzoate as hydrophilic and hydrophobic side groups (Scheme 24). In vivo drug release studies showed that the prepared micelles can prolong drug concentration in blood plasma. ${ }^{137}$ Another attempt in this series was done by replacing ethyl tryptophan in place of ethyl-4-amino benzoate to the polyphosphazene chain while keeping the $\operatorname{poly}(N$-isopropylacrylamide) as the other substituent. ${ }^{138}$ Polyphosphazene with an anti-cancer drug, oxaliplatin, combined with 2hydroxyethoxy-diethylmalate and PEG on other side of the backbone, was synthesized, which, on self-assembly, converted into micelles with a mean diameter of $130 \mathrm{~nm} .{ }^{139}$ These micelles are found to have increased bioavailability and selectivity to the target as compared to oxaliplatin alone.

\subsection{Polyphosphazene blends with other polymers}

Polymers can be mixed physically in different proportions to prepare blends. To prepare the blends, polymers are dissolved in suitable solvents and mixed together, followed by evaporation of the solvents. This results in some physical interactions such as hydrogen bonding and van der Waals forces. In most cases, properties of blends change as compared to individual polymers. Blends can also be further modified into microspheres, fibers and hydrogels.

Three novel PPZs were synthesized and converted into 25\%, $50 \%$ and $75 \%(\mathrm{w} / \mathrm{w}$ ) blends (blends A, B and C) with poly(methyl methacrylate) by physical mixing in chloroform, as shown in 

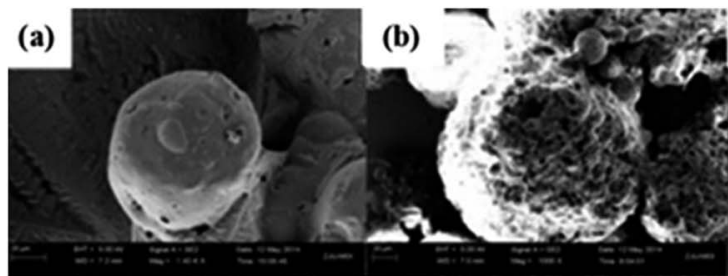

(c)

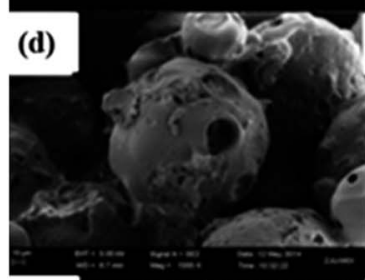

(e)

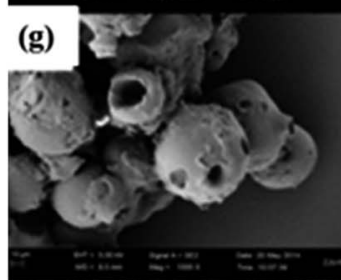

\section{(h)}
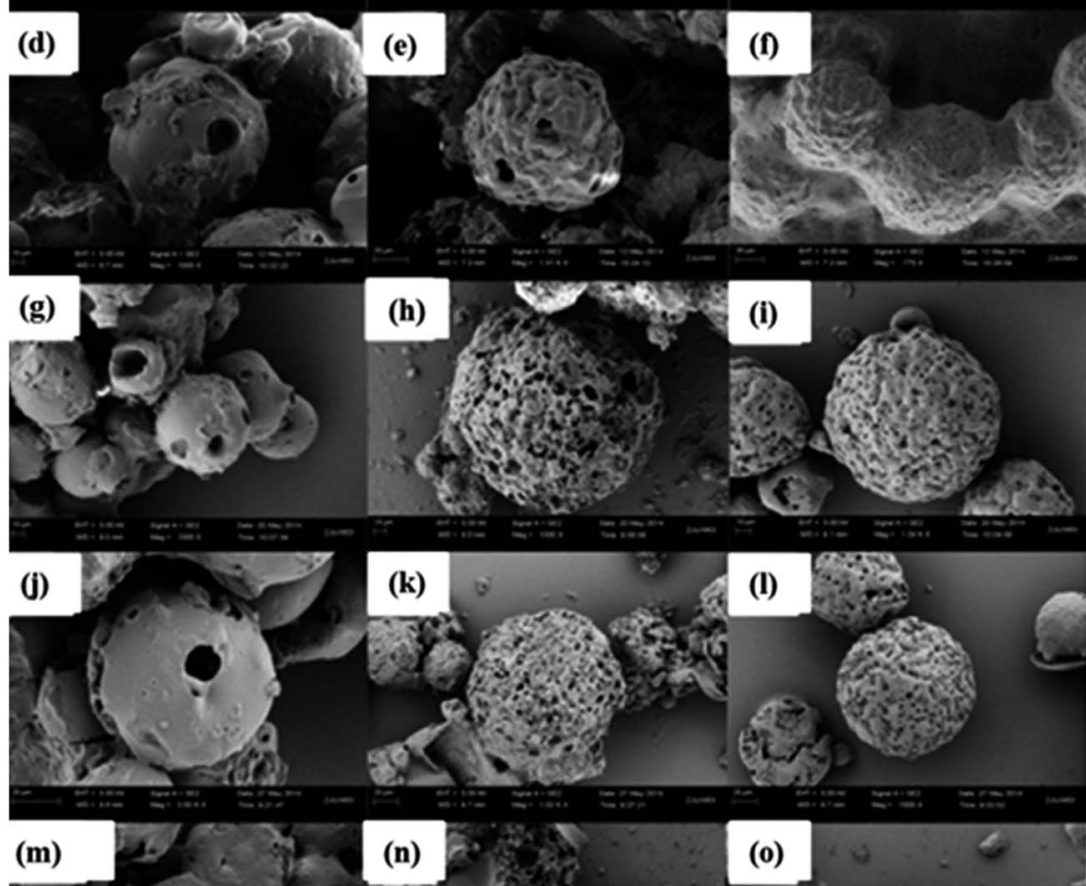

(n)
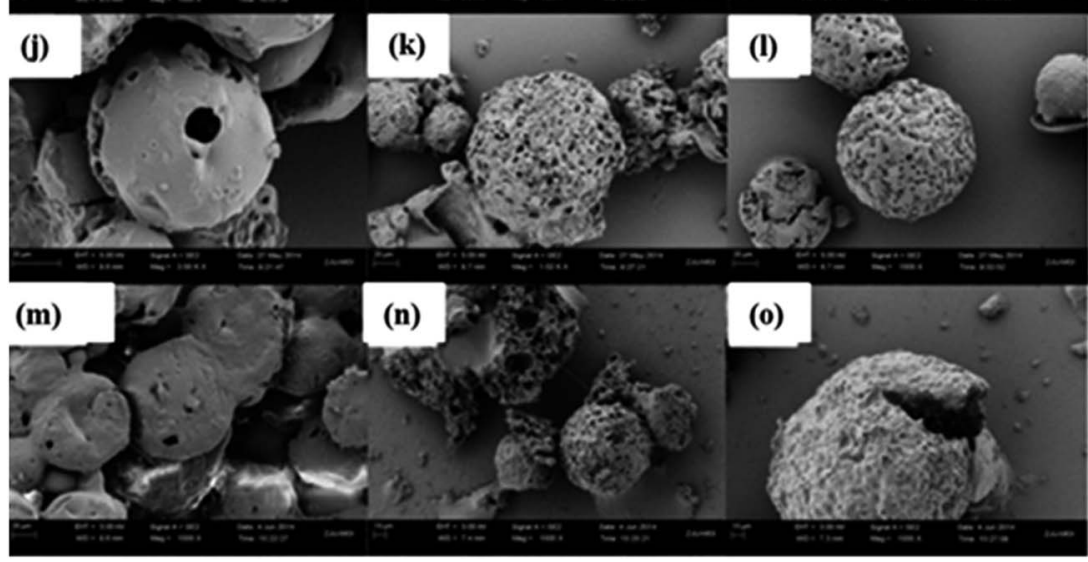

Fig. 12 SEM images showing the effect of degradation of blend microspheres over a period of five weeks (top to bottom) ${ }^{140}$ Copyright 2015 , Elsevier B.V.

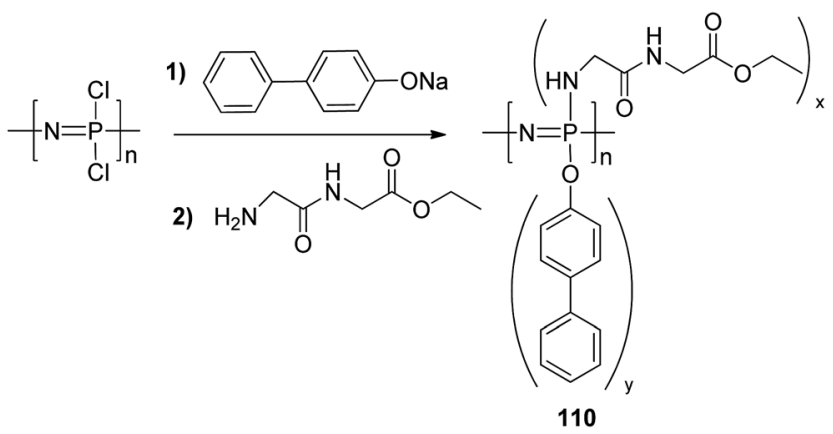

Scheme 25 Synthesis of poly[(glycine ethyl glycinato) ${ }_{1}($ phenyl phenoxy) ${ }_{1}$ phosphazene]. ${ }^{141}$

Fig. 11. The 75\% blends of these three PPZs were further fabricated into microspheres, and sustained release of hydrophobic drugs was evaluated. Microsphere of blend A and $\mathrm{C}$ showed good results of sustained release up to $7.7 \%$ and $20 \%$, while blend C showed more than $90 \%$ release within forty hours (Fig. 12). It was also found that degradation and drug release are related to percentage water retention. ${ }^{\mathbf{1 4 0}}$

Due to their biocompatibility and degradation, polyphosphazene-polyester blend systems are gaining much interest in drug delivery and tissue engineering applications. PPZs with glycylglycine ethyl ester-co-phenylphenoxy groups in 50 : 50 ratio were synthesized as previously reported. ${ }^{5}$ The glycylglycine ethyl ester was attached to enhance the hydrophilic nature of polyphosphazene containing the phenylphenoxy side group (Scheme 25). Blends of these polymers with poly(lactic acid-co-glycolic acid) (PLAGA) in $25: 75$ and $50: 50$ ratios yielded matrix 1 and matrix 2, respectively. The degradation order for these blend systems, in PBS at body temperature for 12 weeks, is matrix $2<$ matrix $1<$ PLAGA (Fig. 13). Both blends were also tested in rat to check their non-toxic nature so these blends can be used for biomedical applications. ${ }^{141}$

The same author studied the unique mechanism of degradation for the polymer (112) blend with PLAGA (50:50). 

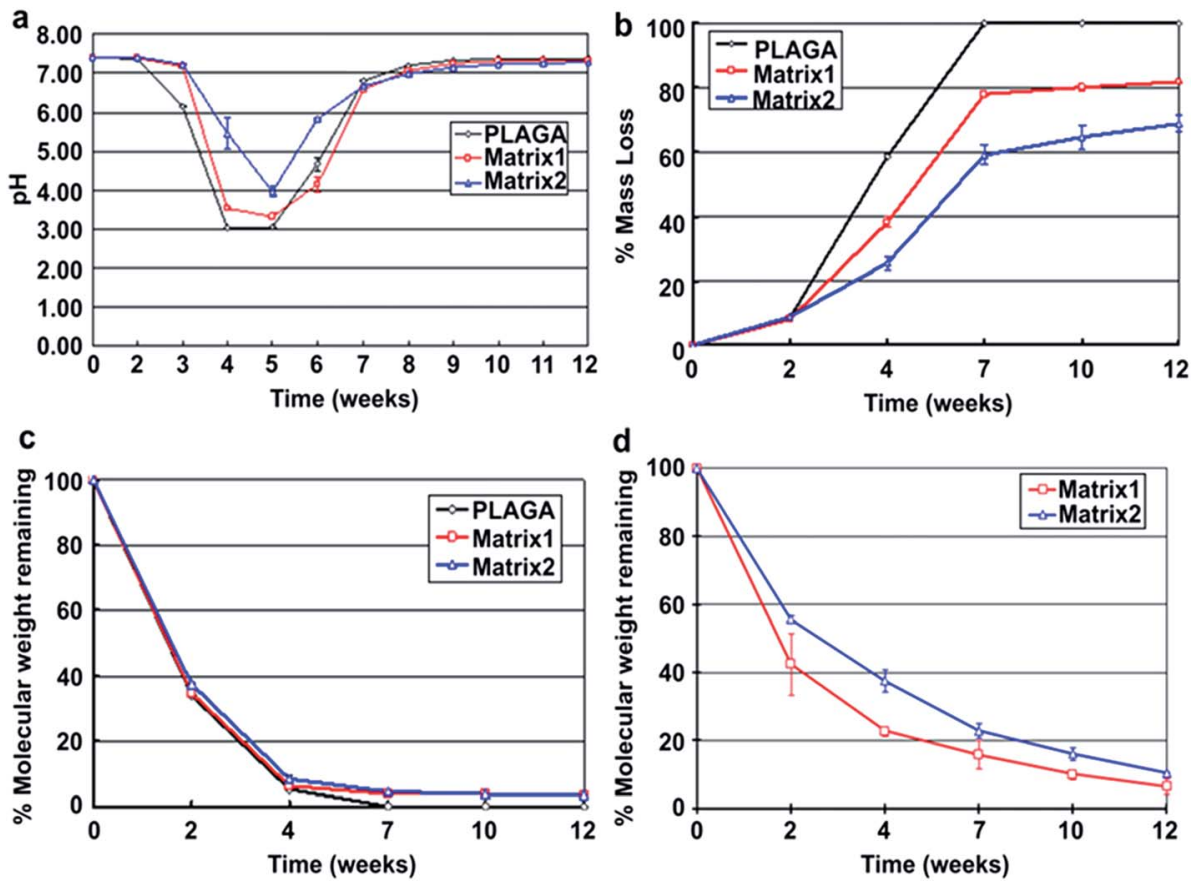

Fig. 13 Degradation profiles of blends and pristine PLAGA in pH 7.4 PBS at $37^{\circ} \mathrm{C}$ over 12 weeks. (a) pH change, (b) percentage of mass loss, (c) percentage of PLAGA molecular weight remaining in the blends and pristine PLAGA over 12 weeks in PBS, (d) percentage of polyphosphazene molecular weight remaining in the blends over 12 weeks in PBS. ${ }^{141}$ Copyright 2010, Elsevier Ltd.

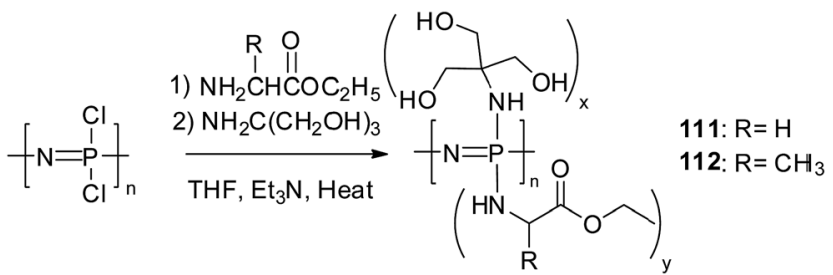

Scheme 26 Synthesis of co-substituted polyphosphazenes with THAM and amino acid (113-114). ${ }^{144}$
Hydrophilic glycylglycine dipeptide encouraged the hydrogen bonding with PLAGA. During degradation, this blend produced 3D spaces which were filled with polyphosphazene spheres formed by self-assembly, showing a porous morphology. ${ }^{\mathbf{1 4 2}}$ This polymer has also been used to fabricate fibers by electrospinning. Length and diameters of these fibers can be controlled by changing the concentration and ratio of polymers and blends. They also created biomimetic 3D scaffolds with an open central cavity. ${ }^{143}$

PPZs with the side groups of tris(hydroxymethyl)amino methane (THAM) and ethyl esters of glycine and alanine have<smiles>[R]C(N)C(=O)OCC</smiles>

Scheme 27 Synthesis of poly[(amino ethyl ester) $)_{x}(\text { choline chloride) })_{y}$ phosphazene] using two different routes: (a) sodium hydride (NaH) (113116), (b) cesium carbonate $\left(\mathrm{Cs}_{2} \mathrm{CO}_{3}\right)(117-120){ }^{145}$ 


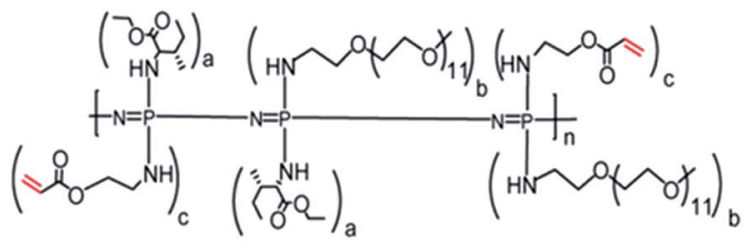

(V) $a+b+c=2$ $\cap$

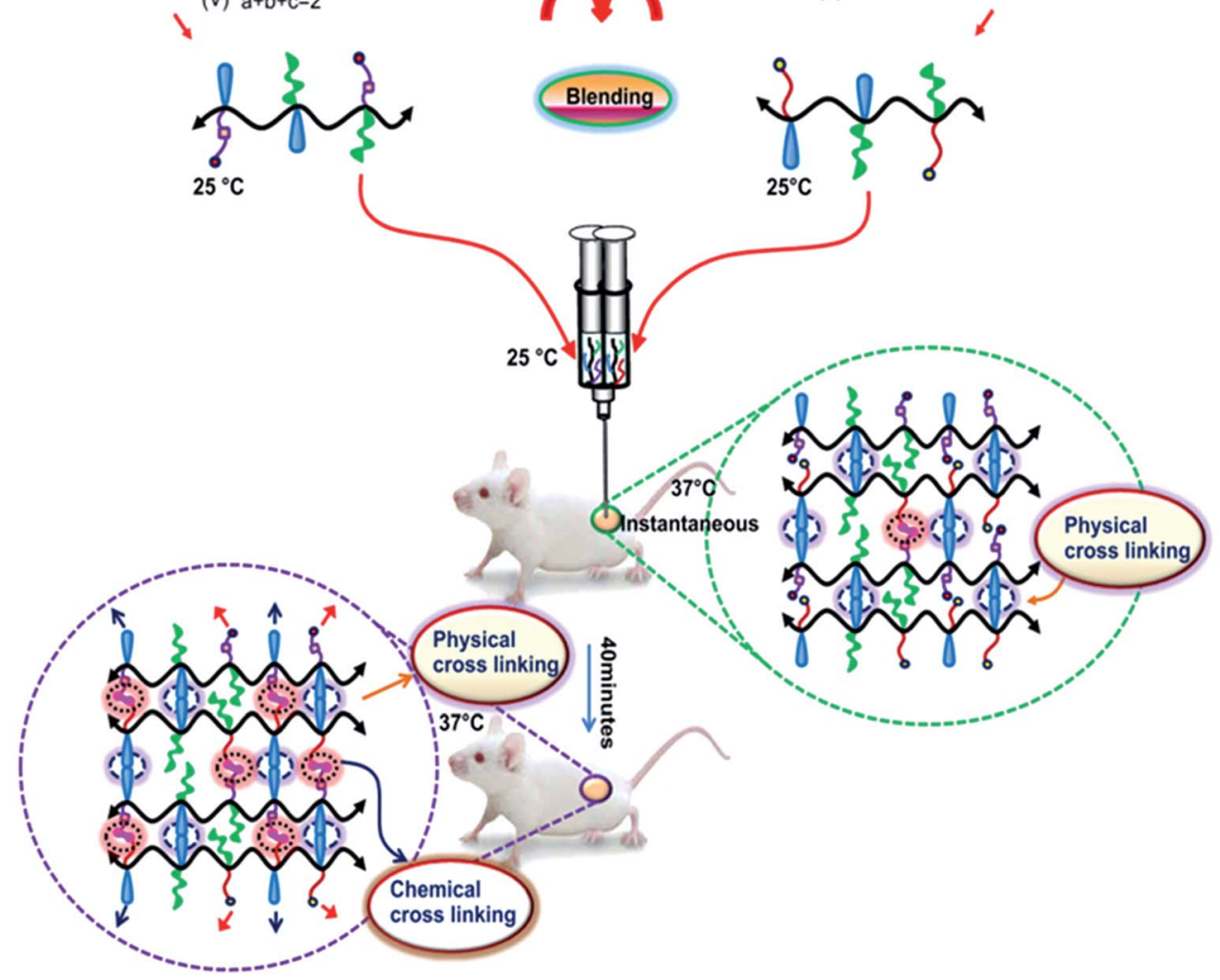

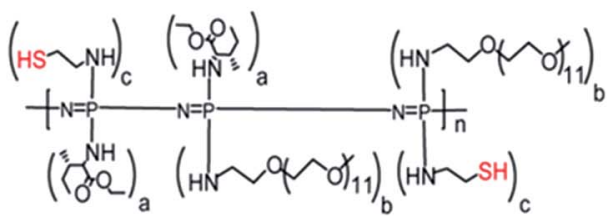

(V) $a+b+c=2$

Fig. 14 Polyphosphazene-based blend hydrogel and demonstration of physical and chemical cross-linking after injection in mice. ${ }^{146}$ Copyright 2010, Elsevier Ltd.

been synthesized (Scheme 26), and then, blends of these polymers with poly(lactic acid-co-glycolic acid) (PLAGA) in (50:50) and $(85: 15)$ ratios were prepared to check their degradation patterns. THAM can increase the hydrogen bonding between the blended polymers. Hydrolytic studies of polyphosphazene: PLAGA (50:50) blends showed that PLAGA contents degraded more rapidly than synthesized PPZ and are safer to use in living organisms, as indicated by cell osteoblast studies. ${ }^{144}$ Synthetic routes also play a role in forming different natures of polymers and their blending behavior. Choline chloride, a biological buffer, has been attached as a side group in PPZs along with four different amino acids using two different methods.

Using the sodium hydride route of synthesis, low-cholinecontent PPZs have been prepared as compared to the cesium carbonate route, which produces high-choline-content PPZs (Scheme 27). The synthesized polymers were then mixed with PLAGA in ratios of $(50: 50)$ and $(85: 15)$ to produce blends that show differences in miscibility. The polymer synthesized through sodium hydride route was more miscible as compared to the polymer synthesized through cesium carbonate route.
After degradation, the blends produce near-neutral medium and showed non-toxic nature. ${ }^{145}$

An interesting work has been done to eradicate the basic problems faced with injectable drug delivery systems. A biocompatible, thermosensitive and water-soluble polyphosphazene blending system has been designed, in which two different PPZs with active thiolated and acrylated functional groups have been synthesized and blended (Fig. 14). At low temperature, it is in solution form, while at body temperature, it is converted into hydrogel form by developing both physical interactions such as hydrogen bonding and chemical interactions such as cross-linking between thiolated and acrylated groups. ${ }^{146}$ Blending of poly[(ethylglycinato)(methoxyethoxyethoxy)phosphazene] with PLAGA in the ratios of $25: 75$ and $50: 50$ was investigated to produce biodegradable materials of high strength that were also biocompatible, as shown by rat osteoblast cell experiment. ${ }^{147}$ Blend formation was affected by the nature of polymers, ratio of mixing and solvents used, and it was checked by the use of SEM and $T_{g}$ values. Sometimes, the blends are completely miscible, having no phase separation, while sometimes, they are partially miscible or completely immiscible depending upon these factors. ${ }^{18,149}$ 
Miscibility of blends was demonstrated by producing PPZs with ethyl glycinate and ethyl glycylglycinate in five different ratios. They were again blended with PLAGA to produce biomaterials of diverse properties. Poly[(ethyl glycinato) ${ }_{0.5}$ (glycine ethyl glycinato) ${ }_{1.5}$ phosphazene] formed miscible blends with PLAGA and showed greater degradation time as compared to the individual polymers. Glass transition temperature of the blend was lower than that of parent polymers, which shows the presence of interactions between them. ${ }^{150}$

\section{Polyphosphazenes towards clinical development}

In order to approve PPZs as functional biological material, various areas of drug delivery have been concentrated and different experiments have been performed. These include in vivo experiments for the successful delivery of anti-cancer drugs. Chun et al. have developed PPZ-based conjugates of doxorubicin and paclitaxel drug and checked their anti-cancer ability against SNU-601 human gastric cancer cells and HSC45M2 human gastric cancer cells, respectively, which were implanted in nude mice. ${ }^{\mathbf{5 4 9 6}}$ These anti-cancer drug delivery systems inhibited the tumor growth effectively for 30 days as compared to free drugs, suggesting these conjugates as potential candidates for clinical trials. Similarly, Cho et al. have also developed an injectable delivery system for 2-methoxyestradiol for breast cancer therapy based on thermosensitive PPZs. ${ }^{151}$ By monitoring tumor volume and CD31 immuno-histochemical staining in mouse orthotopic breast tumor (MDAMB-231) model, it was found that the hydrogel containing a relatively low concentration $\left(15 \mathrm{mg} \mathrm{kg}^{-1}\right)$ of 2 -ME showed improved antitumor and antiangiogenic activity. In other attempts, the same authors have also developed injectable poly(organophosphazene)s for the delivery of silibinin (SIL) and camptothecin (CPT) and performed in vitro and in vivo antitumor activity studies. For in vitro studies, the drug-polymer conjugate hydrogel of CPT showed good anti-tumor activity against four different human cancer cell lines and is also more effective for in vivo studies for up to 15 days against HT-29 colon cancer cell-xerografted mice as compared to CPT alone. ${ }^{47}$ Similarly, SIL in solution form was injected into alreadyimplanted HT-29 colon cancer cell line and showed very effective tumor inhibition. ${ }^{95}$

The more focused area of PPZs towards clinical development is the delivery of vaccines and immunoadjuvants. Pike corporation, based in Korea, has been developing an injectable drugdelivery system based on PPZ block copolymers with thermoreversible properties. ${ }^{152}$ PPZs have been used as carriers for adjuvants and vaccine antigens and also add their mild adjuvant activity. However, when combined with immune stimulators such as CpG, ODN or PolyIC, these molecules can form complexes that become highly immunogenic. ${ }^{153,154}$ One of the most considered PPZ polyelectrolytes, poly[di(carboxylatophenoxy)phosphazene] (PCPP), has been used in phase I clinical study, which was carried out with the influenza vaccine on both young and elderly adults (a total of 96 subjects). ${ }^{155}$
Three different doses, comprising 100, 200 and $500 \mu \mathrm{g}$ of PCPP, were injected and followed for 12 months. The strongest response was observed for the $500 \mu \mathrm{g}$ dose against all three strains of influenza virus, especially in young subjects. PCPP was also inspected in clinical trials through oligomeric HIV-1 Gp160mn/LAI-2 vaccine in HIV-seronegative volunteers from the United States, showing the long-term safety profile of the adjuvant. ${ }^{156,157}$ Another publication also cites the use of PCPP for boost immunization in HIV vaccine clinical trials. ${ }^{\mathbf{1 5 8}}$

Most recently, a phase 1 clinical trial was conducted in which 19 volunteers received oligomeric glycoprotein 160 from HIV strains MN and LAI-2 (ogp160 MN/LAI-2) with variations in dose $(25,50,100 \mu \mathrm{g})$ and either polyphosphazene or alum adjuvant (part A). In part B, 72 volunteers received either placebo $(n=12)$ or recombinant canarypox virus expressing HIV antigens (ALVACHIV [vCP205]) with different doses and schedules of ogp160 MN/ LAI-2 in PPZ or alum $(n=60)$. In both parts, volunteers receiving the PPZ adjuvant produced good response in the neutralization of tier 2 viruses. ${ }^{159}$ In another study, Mishal et al. evaluated the adjuvant activity of poly[di(sodium carboxylatoethylphenoxy)] phosphazene (PCEP) and avian beta defensin (ABD) in a vaccine formulation containing inactivated fowl adenovirus (FAdV) serotype $8 \mathrm{~b}$ administered in ovo. The results presented that a mixture of PCEP and inactivated virus can induce a strong and long-term antibody response. ${ }^{\mathbf{1 6 0}}$ Also, substantial improvement in the expression of genes such as IFN- $\gamma$, IFN- $\alpha$, IL-12(p40) and IL-6 by PCEP reveals its ability to produce immune response, which is beneficial for the development of in ovo vaccines.

\section{Conclusion and future prospects}

PPZ is an emerging class of polymers whose flexibility in properties, biocompatibility and biodegradability are very prominent. Their hydrophilic/hydrophobic nature and degradation behavior can be tuned by changing the side groups. Amongst the most commonly used side groups, ethers provide hydrophilic nature and result in water-soluble PPZs. Amino acid esters have a range of effects-from hydrophilic to hydrophobic-depending on the nature of the amino acid used, while the aromatic side groups are mostly hydrophobic in nature and result in higher $T_{\mathrm{g}}$ values of the PPZs. Mostly, PPZs are prepared by a combination of hydrophobic and hydrophilic side groups, resulting in polymers with balanced properties. Although the synthesis process is a bit difficult and demands care, the incorporation of PPZs in drugdelivery applications is mounting continuously, and many different drug carriers are being developed for the safe and longterm delivery of therapeutic drugs.

Although researchers have tried to attach substituents of all types, there is still a lot to be explored as the flexibility in the PPZ backbone offers great opportunities. To date, the main focus has been on the substituents that contain an amino or hydroxyl group for attachment to the PPZ main chain. However, the effects of those substituents containing other attachment sites, like $-\mathrm{SH},-\mathrm{BH}_{2}$, carbonyl carbon etc., still need to be explored, especially in the field of drug delivery. Furthermore, it is possible to attach such linking atoms other than $\mathrm{N}$ and $\mathrm{O}$, and how they will affect the properties of PPZs is still a question. The 
blending of PPZ with other polymers and different formulations into micro/nano fibers, microspheres, micelles, polymersomes and a lot more is still inviting researchers to explore new dimensions. The clinical development of PPZ is not so established, which is very important for the practical implementation of PPZ as a biomaterial.

\section{References}

1 R. Barbucci, Integrated biomaterials science, Springer, New York, 2002.

2 D. G. Castner and B. D. Ratner, Surf. Sci., 2002, 500, 28-60. 3 D. J. Apple, Sir harold ridley and his fight for sight: He changed the world so that we may better see it, Slack, 2006.

$4 \mathrm{~J}$. Tsung and D. J. Burgess, in Fundamentals and applications of controlled release drug delivery, ed. J. Siepmann, R. A. Siegel and M. J. Rathbone, Springer, 233 Spring Street, New York, Ny 10013, United States, 2012, pp. 107-123, DOI: 10.1007/978-1-4614-0881-9_5.

$5 \mathrm{H}$. R. Allcock, Chemistry and applications of polyphosphazenes, Wiley, New York, 2003.

6 H. R. Allcock, Dalton Trans., 2016, 45, 1856-1862.

7 C. T. Laurencin, M. E. Norman, H. M. Elgendy, S. F. ElAmin, H. R. Allcock, S. R. Pucher and A. A. Ambrosio, J. Biomed. Mater. Res., 1993, 27, 963-973.

8 C. T. Laurencin, S. F. El-Amin, S. E. Ibim, D. A. Willoughby, M. Attawia, H. R. Allcock and A. A. Ambrosio, J. Biomed. Mater. Res., 1996, 30, 133-138.

9 F. Langone, S. Lora, F. M. Veronese, P. Caliceti, P. P. Parnigotto, F. Valenti and G. Palma, Biomaterials, 1995, 16, 347-353.

10 F. M. Veronese, F. Marsilio, S. Lora, P. Caliceti, P. Passi and P. Orsolini, Biomaterials, 1999, 20, 91-98.

11 A. K. Andrianov and L. G. Payne, Adv. Drug Delivery Rev., 1998, 34, 155-170.

12 A. K. Andrianov and L. G. Payne, Adv. Drug Delivery Rev., 1998, 31, 185-196.

13 S. Lakshmi, D. S. Katti and C. T. Laurencin, Adv. Drug Delivery Rev., 2003, 55, 467-482.

14 M. Akram, L. Wang, H. Yu, W. A. Amer, H. Khalid, N. M. Abbasi, Y. Chen, M. Saleem, Zain -ul-Abdin and R. Tong, Prog. Polym. Sci., 2014, 39, 1987-2009.

15 R. De Jaeger and M. Gleria, Prog. Polym. Sci., 1998, 23, 179276.

16 M. Gleria and R. De Jaeger, in New aspects in phosphorus chemistry v, ed. J. P. Majoral, 2005, vol. 250, pp. 165-251.

17 H. R. Allcock, J. Inorg. Organomet. Polym. Mater., 2006, 16, 277-294.

18 H. R. Allcock and R. L. Kugel, J. Am. Chem. Soc., 1965, 87, 4216-4217.

19 H. R. Allcock and R. L. Kugel, Inorg. Chem., 1966, 5, 17161718.

20 H. R. Allcock, R. L. Kugel and K. J. Valan, Inorg. Chem., 1966, 5, 1709-1715.

21 J. H. L. Crommen, E. H. Schacht and E. H. G. Mense, Biomaterials, 1992, 13, 601-611.
22 Y. S. Sohn, Y. H. Cho, H. Baek and O.-S. Jung, Macromolecules, 1995, 28, 7566-7568.

23 H. R. Allcock, J. E. Gardner and K. M. Smeltz, Macromolecules, 1975, 8, 36-42.

24 Y. Cho, Y. S. Sohn and M.-J. Jun, J. Polym. Sci., Part A: Polym. Chem., 1993, 31, 3397-3403.

25 M. S. Sennett, G. L. Hagnauer, R. E. Singler and G. Davies, Macromolecules, 1986, 19, 959-964.

26 S. Ganapathiappan, K. S. Dhathathreyan and S. S. Krishnamurthy, Macromolecules, 1987, 20, 1501-1505.

27 A. M. Amin, L. Wang, H. Yu, W. A. Amer, J. Gao, Y. Tai, J. Huo, Y. Zhang and L. Zhang, J. Macromol. Sci., Part A: Pure Appl. Chem., 2011, 48, 937-946.

28 A. K. Andrianov, J. P. Chen and M. P. LeGolvan, Macromolecules, 2004, 37, 414-420.

29 G. A. Carriedo, F. J. G. Alonso and A. Presa-Soto, Eur. J. Inorg. Chem., 2003, 4341-4346, DOI: 10.1002/ ejic. 200300407.

30 A. N. Mujumdar, S. G. Young, R. L. Merker and J. H. Magill, Macromolecules, 1990, 23, 14-21.

31 G. A. Carriedo, F. L. G. Alonso, P. Gomez-Elipe, J. I. Fidalgo, J. L. G. Alvarez and A. Presa-Soto, Chem.-Eur. J., 2003, 9, 3833-3836.

32 H. R. Allcock, C. A. Crane, C. T. Morrissey, J. M. Nelson, S. D. Reeves, C. H. Honeyman and I. Manners, Macromolecules, 1996, 29, 7740-7747.

33 C. H. Honeyman, I. Manners, C. T. Morrissey and H. R. Allcock, J. Am. Chem. Soc., 1995, 117, 7035-7036.

34 Y. Zhang, K. Huynh, I. Manners and C. A. Reed, Chem. Commun., 2008, 494-496, DOI: 10.1039/b713933k.

35 N. R. Krogman, L. Steely, M. D. Hindenlang, L. S. Nair, C. T. Laurencin and H. R. Allcock, Macromolecules, 2008, 41, 1126-1130.

36 H. R. Allcock, S. D. Reeves, J. M. Nelson, C. A. Crane and I. Manners, Macromolecules, 1997, 30, 2213-2215.

37 Y. Chang, S. C. Lee, K. T. Him, C. Him, S. D. Reeves and H. R. Allcock, Macromolecules, 2001, 34, 269-274.

38 H. R. Allcock, R. Prange and T. J. Hartle, Macromolecules, 2001, 34, 5463-5470.

39 Y. Y. Chang, R. Prange, H. R. Allcock, S. C. Lee and C. Kim, Macromolecules, 2002, 35, 8556-8559.

40 H. R. Allcock, S. Y. Cho and L. B. Steely, Macromolecules, 2006, 39, 8334-8338.

41 H. R. Allcock, E. S. Powell, A. E. Maher, R. L. Prange and C. R. de Denus, Macromolecules, 2004, 37, 3635-3641.

42 H. R. Allcock, J. M. Nelson, R. Prange, C. A. Crane and C. R. de Denus, Macromolecules, 1999, 32, 5736-5743.

43 H. R. Allcock, E. S. Powell, Y. Y. Chang and C. Kim, Macromolecules, 2004, 37, 7163-7167.

44 H. R. Allcock and N. L. Morozowich, Polym. Chem., 2012, 3, 578-590.

45 J. L. Nichol, I. T. Hotham and H. R. Allcock, Polym. Degrad. Stab., 2014, 109, 92-96.

46 A. Singh, N. R. Krogman, S. Sethuraman, L. S. Nair, J. L. Sturgeon, P. W. Brown, C. T. Laurencin and H. R. Allcock, Biomacromolecules, 2006, 7, 914-918. 
47 J.-K. Cho, C. Chun, H.-J. Kuh and S.-C. Song, Eur. J. Pharm. Biopharm., 2012, 81, 582-590.

48 A. Linhardt, M. König, W. Schöfberger, O. Brüggemann, A. Andrianov and I. Teasdale, Polymers, 2016, 8, 161.

49 J. H. L. Crommen, E. H. Schacht and E. H. G. Mense, Biomaterials, 1992, 13, 511-520.

50 J. L. Nichol and H. R. Allcock, Eur. Polym. J., 2015, 62, 214221.

51 J. L. Nichol, N. L. Morozowich and H. R. Allcock, Polym. Chem., 2013, 4, 600-606.

52 B. D. Ratner and S. J. Bryant, Annu. Rev. Biomed. Eng., 2004, 6, 41-75.

53 O. Oredein-McCoy, N. R. Krogman, A. L. Weikel, M. D. Hindenlang, H. R. Allcock and C. T. Laurencin, J. Microencapsulation, 2009, 26, 544-555.

54 C. Chun, S. M. Lee, C. W. Kim, K. Y. Hong, S. Y. Kim, H. K. Yang and S. C. Song, Biomaterials, 2009, 30, 47524762.

55 S. Wilfert, A. Iturmendi, W. Schoefberger, K. Kryeziu, P. Heffeter, W. Berger, O. Bruggemann and I. Teasdale, $J$. Polym. Sci., Part A: Polym. Chem., 2014, 52, 287-294.

56 K. B. Gudasi, R. S. Vadavi, N. B. Shelke, M. Sairam and T. M. Aminahbavi, React. Funct. Polym., 2006, 66, 11491157.

57 H. R. Allcock, S. R. Pucher and A. G. Scopelianos, Macromolecules, 1994, 27, 1-4.

58 H. R. Allcock, D. L. Olmeijer and S. J. M. O'Connor, Macromolecules, 1998, 31, 753-759.

59 J. M. G. Cowie and K. Sadaghianizadeh, Makromol. Chem., Rapid Commun., 1988, 9, 387-391.

60 A. K. Andrianov, A. Marin and J. P. Chen, Biomacromolecules, 2006, 7, 394-399.

61 A. K. Andrianov, D. P. DeCollibus, H. A. Gillis, H. H. Kha, A. Marin, M. R. Prausnitz, L. A. Babiuk, H. Townsend and G. Mutwiri, Proc. Natl. Acad. Sci. U. S. A., 2009, 106, 18936-18941.

62 M. Luisa Valenzuela and G. A. Carriedo, Polymer, 2011, 52, 2157-2162.

63 S. K. Kwon, Bull. Korean Chem. Soc., 2000, 21, 969-972.

64 H. R. Allcock and S. R. Pucher, Macromolecules, 1991, 24, 23-34.

65 J. L. Nichol, N. L. Morozowich, T. E. Decker and H. R. Allcock, J. Polym. Sci., Part A: Polym. Chem., 2014, 52, 2258-2265.

66 S. Sethuraman, L. S. Nair, S. El-Amin, R. Farrar, M. T. Nguyen, A. Singh, H. R. Allcock, Y. E. Greish, P. W. Brown and C. T. Laurencin, J. Biomed. Mater. Res., Part A, 2006, 77, 679-687.

67 Y. J. Jun, J. H. Kim, S. J. Choi, H. J. Lee, M. J. Jun and Y. S. Sohn, Bioorg. Med. Chem. Lett., 2007, 17, 2975-2978.

68 R. Haag and F. Kratz, Angew. Chem., Int. Ed. Engl., 2006, 45, 1198-1215.

69 H. Maeda, J. Wu, T. Sawa, Y. Matsumura and K. Hori, J. Controlled Release, 2000, 65, 271-284.

70 R. Duncan, Nat. Rev. Drug Discovery, 2003, 2, 347-360.

71 H. Ringsdorf, J. Polym. Sci., Part C: Polym. Symp., 1975, 135153.
72 K. J. Haxton and H. M. Burt, J. Pharm. Sci., 2009, 98, 22992316.

73 Z. Tian, Y. Zhang, X. Liu, C. Chen, M. J. Guiltinan and H. R. Allcock, Polym. Chem., 2013, 4, 1826.

74 G. L. Y. Woo, M. L. Yang, H. Q. Yin, F. Jaffer, M. W. Mittleman and J. P. Santerre, J. Biomed. Mater. Res., 2002, 59, 35-45.

75 I. Teasdale, M. Waser, S. Wilfert, H. Falk and O. Brueggemann, Monatsh. Chem., 2012, 143, 355-360.

76 N. L. Morozowich, J. L. Nichol, R. J. Mondschein and H. R. Allcock, Polym. Chem., 2012, 3, 778-786.

77 S. Kumar, R. K. Singh, R. Sharma, R. S. R. Murthy and T. R. Bhardwaj, Eur. J. Pharm. Sci., 2015, 66, 123-137.

78 H. R. Allcock and W. E. Krause, Macromolecules, 1997, 30, 5683-5687.

79 I. Teasdale, S. Wilfert, I. Nischang and O. Brueggemann, Polym. Chem., 2011, 2, 828-834.

80 R. Sharma, R. K. Rawal, M. Malhotra, A. K. Sharma and T. R. Bhardwaj, Bioorg. Med. Chem., 2014, 22, 1104-1114.

81 H. Henke, K. Kryeziu, J. Banfić, S. Theiner, W. Körner, O. Brüggemann, W. Berger, B. K. Keppler, P. Heffeter and I. Teasdale, Macromol. Biosci., 2016, 16, 1239-1249.

82 M. W. Tibbitt and K. S. Anseth, Biotechnol. Bioeng., 2009, 103, 655-663.

83 A. S. Hoffman, Adv. Drug Delivery Rev., 2002, 54, 3-12.

84 D. A. Cardoso, J. J. J. P. van den Beucken, L. L. H. Both, J. Bender, J. A. Jansen and S. C. G. Leeuwenburgh, J. Biomed. Mater. Res., Part A, 2014, 102, 808-817.

85 C. Karakasyan, M. Legros, S. Lack, F. Brunel, P. Maingault, G. Ducouret and D. Hourdet, Biomacromolecules, 2010, 11, 2966-2975.

86 B. H. Lee, Y. M. Lee, Y. S. Sohn and S. C. Song, Macromolecules, 2002, 35, 3876-3879.

87 M.-R. Park, C. Chun, S.-W. Ahn, M.-H. Ki, C.-S. Cho and S.-C. Song, J. Controlled Release, 2010, 147, 359-367.

88 C. Chun, H. J. Lim, K.-Y. Hong, K.-H. Park and S.-C. Song, Biomaterials, 2009, 30, 6295-6308.

89 J.-Y. Yoon, K.-H. Park and S.-C. Song, J. Biomater. Sci., Polym. Ed., 2007, 18, 1181-1193.

90 J. I. Kim, C. Chun, B. Kim, J. M. Hong, J.-K. Cho, S. H. Lee and S.-C. Song, Biomaterials, 2012, 33, 218-224.

91 Y.-C. Qian, P.-C. Chen, G.-J. He, X.-J. Huang and Z.-K. Xu, Molecules, 2014, 19, 9850-9863.

92 Z. Huang, X. Liu, S. Chen, Q. Lu and G. Sun, Polym. Chem., 2015, 6, 143-149.

93 Z. C. Tian, C. Chen and H. R. Allcock, Macromolecules, 2013, 46, 2715-2724.

94 J.-K. Cho, J. M. Hong, T. Han, H.-K. Yang and S.-C. Song, J. Drug Targeting, 2013, 21, 564-573.

95 J.-K. Cho, J. W. Park and S.-C. Song, J. Pharm. Sci., 2012, 101, 2382-2391.

96 C. Chun, S. M. Lee, S. Y. Kim, H. K. Yang and S. C. Song, Biomaterials, 2009, 30, 2349-2360.

97 J. A. Floyd, A. Galperin and B. D. Ratner, Adv. Drug Delivery Rev., 2015, 91, 23-37.

98 H. Ozay and O. Ozay, Colloids Surf., A, 2014, 450, 99-105. 
99 J. Koehler, S. Kuehl, H. Keul, M. Moeller and A. Pich, J. Polym. Sci., Part A: Polym. Chem., 2014, 52, 527-536.

100 J. L. Brown, L. S. Nair and C. T. Laurencin, J. Biomed. Mater. Res., Part B, 2008, 86, 396-406.

101 M. Wilhelm, C. L. Zhao, Y. C. Wang, R. L. Xu, M. A. Winnik, J. L. Mura, G. Riess and M. D. Croucher, Macromolecules, 1991, 24, 1033-1040.

102 N. Kumar, M. N. V. Ravikumar and A. J. Domb, Adv. Drug Delivery Rev., 2001, 53, 23-44.

103 P. Caliceti, F. M. Veronese and S. Lora, Int. J. Pharm., 2000, 211, 57-65.

104 A. K. Andrianov and J. P. Chen, J. Appl. Polym. Sci., 2006, 101, 414-419.

105 A. K. Andrianov, J. Chen and L. G. Payne, Biomaterials, 1998, 19, 109-115.

106 F. M. Veronese, F. Marsilio, P. Caliceti, P. De Filippis, P. Giunchedi and S. Lora, J. Controlled Release, 1998, 52, 227-237.

107 R. Prange, S. D. Reeves and H. R. Allcock, Macromolecules, 2000, 33, 5763-5765.

108 H. R. Allcock and R. Prange, Macromolecules, 2001, 34, 6858-6865.

109 G. Riess, Prog. Polym. Sci., 2003, 28, 1107-1170.

110 S. Jain and F. S. Bates, Science, 2003, 300, 460-464.

111 M. Antonietti and S. Förster, Adv. Mater., 2003, 15, 13231333.

112 A. Graff, M. Sauer, P. Van Gelder and W. Meier, Proc. Natl. Acad. Sci. U. S. A., 2002, 99, 5064-5068.

113 C. Nardin, J. Widmer, M. Winterhalter and W. Meier, Eur. Phys. J. E: Soft Matter Biol. Phys., 2001, 4, 403-410.

114 Y. Bae, S. Fukushima, A. Harada and K. Kataoka, Angew. Chem., Int. Ed., 2003, 42, 4640-4643.

115 J. X. Zhang, L. Y. Qiu, Y. Jin and K. J. Zhu, Macromolecules, 2006, 39, 451-455.

116 J. X. Zhang, L. Y. Qiu and K. J. Zhu, Macromol. Rapid Commun., 2005, 26, 1716-1723.

117 J. Y. Chang, P. J. Park and M. J. Han, Macromolecules, 2000, 33, 321-325.

118 F. Fu, H. Xu, Y. Dong, M. He, Z. Zhang, T. Luo, Y. Zhang, X. Hao and C. Zhu, J. Membr. Sci., 2015, 489, 119-128.

119 F. Fu, H. Xu, Y. Dong, M. He, T. Luo, Y. Zhang, X. Hao, T. Ma and C. Zhu, Solid State Ionics, 2015, 278, 58-64.

120 J. F. Gohy, in Block copolymers ii, ed. V. Abetz, SpringerVerlag Berlin, Berlin, 2005, vol. 190, pp. 65-136.

121 K. K. Jette, D. Law, E. A. Schmitt and G. S. Kwon, Pharm. Res., 2004, 21, 1184-1191.

122 A. N. Lukyanov and V. P. Torchilin, Adv. Drug Delivery Rev., 2004, 56, 1273-1289.

123 Y. Matsumura, T. Hamaguchi, T. Ura, K. Muro, Y. Yamada, Y. Shimada, K. Shirao, T. Okusaka, H. Ueno, M. Ikeda and N. Watanabe, Br. J. Cancer, 2004, 91, 1775-1781.

124 S. Eetezadi, S. N. Ekdawi and C. Allen, Adv. Drug Delivery Rev., 2015, 91, 7-22.

125 H. Wang, L. Tang, C. L. Tu, Z. Y. Song, Q. Yin, L. C. Yin, Z. H. Zhang and J. J. Cheng, Biomacromolecules, 2013, 14, 3706-3712.
126 R. R. Wei, L. Cheng, M. Zheng, R. Cheng, F. H. Meng, C. Deng and Z. Y. Zhong, Biomacromolecules, 2012, 13, 2429-2438.

127 Y. L. Wu, W. Chen, F. H. Meng, Z. J. Wang, R. Cheng, C. Deng, H. Y. Liu and Z. Y. Zhong, J. Controlled Release, 2012, 164, 338-345.

128 L. Y. Qiu, X. L. Wu and Y. Jin, Pharm. Res., 2008, 26, 946957.

129 J. X. Zhang, L. Y. Qiu, Y. Jin and K. J. Zhu, J. Biomed. Mater. Res., Part A, 2006, 76, 773-780.

130 J. X. Zhang, L. Y. Qiu, K. J. Zhu and Y. Jin, Macromol. Rapid Commun., 2004, 25, 1563-1567.

131 C. Zheng, L. Qiu, X. Yao and K. Zhu, Int. J. Pharm., 2009, 373, 133-140.

132 L. Y. Qiu and M. Q. Yan, Acta Biomater., 2009, 5, 2132-2141.

133 T. Doan and E. Massarotti, J. Clin. Pharmacol., 2005, 45, 751-762.

134 E. D. Harris, N. Engl. J. Med., 1990, 322, 1277-1289.

135 J. Steinmeyer and Y. T. Konttinen, Adv. Drug Delivery Rev., 2006, 58, 168-211.

136 J. X. Zhang, M. Q. Yan, X. H. Li, L. Y. Qiu, X. D. Li, X. J. Li, Y. Jin and K. J. Zhu, Pharm. Res., 2007, 24, 1944-1953.

137 J. X. Zhang, L. Y. Qiu, Y. Jin and K. J. Zhu, Colloids Surf., B, 2005, 43, 123-130.

138 J. X. Zhang, X. J. Li, L. Y. Qiu, X. H. Li, M. Q. Yan, J. Yi and K. J. Zhu, J. Controlled Release, 2006, 116, 322-329.

139 P. G. Avaji, H. I. Joo, J. H. Park, K. S. Park, Y. J. Jun, H. J. Lee and Y. S. Sohn, J. Inorg. Biochem., 2014, 140, 45-52.

140 M. Akram, H. Yu, L. Wang, H. Khalid, N. M. Abbasi, A. Zain ul, Y. Chen, F. Ren and M. Saleem, Mater. Sci. Eng., C, 2016, 58, 169-179.

141 M. Deng, L. S. Nair, S. P. Nukavarapu, T. Jiang, W. A. Kanner, X. Li, S. G. Kumbar, A. L. Weikel, N. R. Krogman, H. R. Allcock and C. T. Laurencin, Biomaterials, 2010, 31, 4898-4908.

142 M. Deng, L. S. Nair, S. P. Nukavarapu, S. G. Kumbar, T. Jiang, A. L. Weikel, N. R. Krogman, H. R. Allcock and C. T. Laurencin, Adv. Funct. Mater., 2010, 20, 2794-2806.

143 M. Deng, S. G. Kumbar, L. S. Nair, A. L. Weikel, H. R. Allcock and C. T. Laurencin, Adv. Funct. Mater., 2011, 21, 2641-2651.

144 N. R. Krogman, A. L. Weikel, K. A. Kristhart, S. P. Nukavarapu, M. Deng, L. S. Nair, C. T. Laurencin and H. R. Allcock, Biomaterials, 2009, 30, 3035-3041.

145 A. L. Weikel, S. G. Owens, N. L. Morozowich, M. Deng, L. S. Nair, C. T. Laurencin and H. R. Allcock, Biomaterials, 2010, 31, 8507-8515.

146 T. Potta, C. Chun and S. C. Song, Biomaterials, 2010, 31, 8107-8120.

147 M. Deng, L. S. Nair, S. P. Nukavarapu, S. G. Kumbar, J. L. Brown, N. R. Krogman, A. L. Weikel, H. R. Allcock and C. T. Laurencin, J. Biomed. Mater. Res., Part A, 2010, 92, 114-125.

148 M. Deng, L. S. Nair, S. P. Nukavarapu, S. G. Kumbar, T. Jiang, N. R. Krogman, A. Singh, H. R. Allcock and C. T. Laurencin, Biomaterials, 2008, 29, 337-349. 
149 D.-H. Chen, L. Hong, X.-W. Nie, X.-L. Wang and X.-Z. Tang, Eur. Polym. J., 2003, 39, 871-876.

150 N. R. Krogman, A. Singh, L. S. Nair, C. T. Laurencin and H. R. Allcock, Biomacromolecules, 2007, 8, 1306-1312.

151 J.-K. Cho, K.-Y. Hong, J. W. Park, H.-K. Yang and S.-C. Song, J. Drug Targeting, 2011, 19, 270-280.

152 M. V. Chaubal, Polymeric materials for drug delivery applications, Market Report, Techvest LLC, 2003.

153 N. F. Eng, S. Garlapati, V. Gerdts, A. Potter, L. A. Babiuk and G. K. Mutwiri, Curr. Drug Delivery, 2010, 7, 13-20.

154 G. Mutwiri, P. Benjamin, H. Soita, H. Townsend, R. Yost, B. Roberts, A. K. Andrianov and L. A. Babiuk, Vaccine, 2007, 25, 1204-1213.

155 N. B. Le Cam, J. Ronco, A. Francon, C. Blondeau and B. Fanget, Res. Immunol., 1998, 149, 19-23.
156 P. Gilbert, Y.-L. Chiu, M. Allen, D. Lawrence, C. Chapdu, H. Israel, D. Holman, M. Keefer, M. Wolff and S. Frey, Vaccine, 2003, 21, 2933-2947.

157 J. Kim, E. Kirsch, B. Gilliam, N. Michael, T. VanCott, S. Ratto-Kim, J. Cox, R. Nielsen, M. Robb and P. Caudrelier, 37th Annual Meeting of the Infectious Diseases Society of America, 1999, p. 1028.

158 P. Thongcharoen, V. Suriyanon, R. M. Paris, C. Khamboonruang, M. S. de Souza, S. Ratto-Kim, C. Karnasuta, V. R. Polonis, L. Baglyos and R. El Habib, JAIDS, J. Acquired Immune Defic. Syndr., 2007, 46, 48-55.

159 R. J. O'connell, J.-L. Excler, V. R. Polonis, S. Ratto-Kim, J. Cox, L. L. Jagodzinski, M. Liu, L. Wieczorek, J. G. McNeil and R. El-Habib, J. Infect. Dis., 2016, jiw059.

160 M. Sarfraz, M. Suleman, S. K. Tikoo, C. Wheler, A. A. Potter, V. Gerdts and A. Dar, Vaccine, 2017, 35(6), 981-986. 